\title{
Sviluppare la metacognizione nel problem solving: un percorso di ricerca didattica nella scuola secondaria di primo grado
}

\section{Development of metacognition in problem solving: a research work in mathematics education for lower secondary school}

\author{
Daniela Pietrapiana e Stefania Donadio \\ Scuola secondaria di primo grado Don Milani, Istituto Onnicomprensivo annesso \\ al Convitto Nazionale C. Colombo - Genova, Italia \\ 凶pietrapiana@donmilani.wikischool.it, stefania.donadio@donmilani.wikischool.it
}

Sunto / Le difficoltà incontrate dagli studenti nel problem solving (PS) sono da sempre oggetto di studio nella ricerca in didattica della matemati$\mathrm{ca}$, ma solo recentemente l'attenzione si è focalizzata sulla metacognizione e sul suo ruolo.

In una scuola secondaria di primo grado italiana, è stato realizzato un percorso di apprendimento sul PS rivolto al potenziamento della metacognizione, con uso di strumenti scientificamente validati e fruibili dai docenti senza la mediazione o il supporto di esperti.

Vengono descritte e discusse le attività proposte in tre classi terze (campione di 54 alunni), organizzate prevalentemente in lavori di gruppo di studenti suddivisi per livelli di competenza.

Dalla valutazione conclusiva è emerso un miglioramento nelle capacità metacognitive e negli esiti del PS, sebbene il dato sia apparso meno significativo per gli studenti con bisogni educativi speciali (BES).

Parole chiave: metacognizione; problem solving; test standardizzati; valutazione.
Abstract / Research in mathematics education has always concerned the difficulties encountered by students in problem solving (PS); however, attention has only recently been focused on metacognition and its role.

Three 8th grade classes of an Italian lower secondary school have been subjected to an educational path that was designed with the aim of enhancing metacognition in PS and with the use of scientifically validated tools that can be used by teachers without any specific intervention and support of external experts.

The activities proposed to a sample of 54 students (divided into work groups according to their performance level in PS) are here described and discussed.

The final evaluation revealed an improvement in metacognitive abilities and PS outcomes, though results were less significant for students with special educational needs.

Keywords: metacognition; problem solving; standardized tests; evaluation. 


\section{Introduzione}

Da sempre il problem solving (PS) in matematica è considerato una competenza complessa nella sua struttura e difficile da gestire da parte degli insegnanti, per via delle molteplici componenti che entrano in gioco. Tra queste vi sono aspetti espressamente cognitivi, metacognitivi ed emotivo-motivazionali che hanno un impatto notevole sull'apprendimento (Cornoldi, 1995a, 1995b). Per quanto sia essenziale sviluppare negli studenti capacità metacognitive, di fatto mancano materiali e strumenti flessibili e adatti ad essere utilizzati in classe nell'attività quotidiana. Diverse metodologie, infatti, rimangono ad uso esperto dei ricercatori e si rivelano perciò di difficile riproducibilità per gli insegnanti.

Partendo da questi presupposti, è stato realizzato un percorso di ricerca didattica nelle classi terze di una scuola secondaria di primo grado ${ }^{1}$, interamente progettato da insegnanti di matematica, con I'intento di potenziare la metacognizione, valutare il relativo livello di sviluppo e individuare gli effetti sulla performance degli alunni nel PS.

\section{Il concetto di metacognizione}

La concettualizzazione di questo costrutto complesso chiamato metacognizione è da sempre difficile e dibattuta, tanto che, a oltre quarant'anni dalla sua introduzione, gli studiosi non sono ancora riusciti ad accordarsi su una definizione univoca. II termine è stato originariamente coniato da Flavell (1976), per significare tutti quei processi di cognizione sui processi cognitivi, ovvero del pensiero sul pensiero. Pochi anni dopo, Brown (Brown, 1978; Brown \& DeLoache, 1978) ha arricchito questa definizione con i concetti di monitoraggio e controllo, ovvero con tutti quei processi che regolano I'attività cognitiva mentre essa avviene.

Studi successivi si sono focalizzati sulla ricerca di altri aspetti legati alla metacognizione e hanno dato il via a una proliferazione di termini e di definizioni come credenza metacognitiva, consapevolezza metacognitiva, esperienza metacognitiva, feeling of knowing, judgment of learning, teoria della mente, metamemoria, autoregolazione ecc. (un utile approfondimento si trova in Lai, 2011).

Quanto alla sua definizione, risulta complesso anche discriminare i processi che sono esclusivamente cognitivi da quelli che sono puramente metacognitivi: la maggior parte degli studiosi concordano sul fatto che la metacognizione sia una cognizione di grado superiore, un qualcosa che sovrintende, valuta e regola la cognizione: anche se sovrapposti, è possibile quantomeno individuare processi a decisa prevalenza metacognitiva rispetto a quelli meramente cognitivi (Veenman, Van Hout-Wolters \& Afflerbach, 2006).

Per quanto riguarda il PS, è interessante la suddivisione dei processi metacognitivi in online e offline, dove con processi offline si intendono quelli che avvengono al di fuori, cioè prima o dopo, del processo risolutivo, ad esempio l'autovalutazione, mentre con processi online si considerano quelli messi in atto mentre il processo di risoluzione sta avvenendo, ad esempio il monitoraggio e il controllo (Desoete, Roeyers \& De Clercq, 2003; Pintrich, Wolters \& Baxter, 2000; Veenman, 2005). Occorre considerare però che i metodi di valutazione offline sono considerati meno affidabili di quelli online (Veenman \& Van Cleef, 2019) e che lavorare per migliorare i processi metacognitivi offline a tutto

1. Si tratta della scuola secondaria di primo grado Don Milani di Genova, parte dell'Istituto Onnicomprensivo annesso al Convitto Nazionale C. Colombo. La scuola secondaria di primo grado in Italia dura tre anni e corrisponde ai primi tre anni di scuola media nel Canton Ticino. 
tondo comprende interventi ad ampio spettro e di lunga durata che non possono risolversi all'interno di una singola unità didattica (Pietrapiana, 2016). Lavorare per migliorare i processi online comprende tipicamente interventi più situati e riferiti a domini maggiormente specifici, come ad esempio quello del PS.

\subsection{Il problem solving in matematica, una sua definizione e componenti}

Per una definizione di PS in matematica occorre considerare in senso più generale il concetto di competenza e di didattica per competenze, che risulta il contesto di lavoro più appropriato.

Sulla didattica per competenze in tutte le sue fasi, dalla progettazione alla valutazione, troviamo una descrizione completa in Castoldi $(2009,2011,2013)$ che, prendendo spunto da diverse ricerche, delinea una definizione del costrutto di competenza e, in particolare, partendo dal lavoro di Schoenfeld (1992), uno dei principali studiosi del PS e metacognizione in matematica, identifica quattro componenti del PS matematico:

1. le risorse cognitive, ovvero le conoscenze e le abilità necessarie alla risoluzione del problema (ad es. concetto di raggio, formule per calcolare il perimetro, abilità nel fare somme ed effettuare sottrazioni);

2. le euristiche, ovvero la capacità di individuare il problema, di metterlo a fuoco, di rappresentarlo;

3. le capacità strategiche, ovvero le modalità con cui progettare la risposta, monitorare la soluzione, valutare la plausibilità del risultato;

4. il sistema di valori del soggetto, con particolare riguardo alla sua idea di matematica e di se stesso in rapporto alla matematica (Castoldi, 2009).

Occorre considerare che non tutti i problemi che troviamo nei capitoli dei libri di matematica attivano la competenza: molti sono i cosiddetti "problemi di routine", ovvero quegli esercizi in cui è sufficiente applicare una procedura conosciuta e scontata per arrivare alla soluzione. Quando ripetere una procedura non è più sufficiente, occorre accedere alla memoria, individuare situazioni simili, creare rappresentazioni e connessioni, tentare delle soluzioni cercando di prevedere la loro efficacia. Solo a seguito di questa mobilitazione di risorse, la competenza si attiva (Schoenfeld, 1992).

In letteratura sono state proposte diverse classificazioni del processo risolutivo, le quali si differenziano essenzialmente per il numero delle fasi che lo compongono; in alcuni modelli due o più fasi possono essere raggruppate in una, ma tutti concordano nell'individuare una fase iniziale di comprensione del problema, una fase di pianificazione, una di risoluzione vera e propria ed una di controllo. In Figura 1 proponiamo, tra le tante, una schematizzazione tratta da un lavoro di Robertson (2016) che ha il pregio di evidenziare la circolarità del processo, il quale può essere percorso e ripercorso più volte in entrambe le direzioni. Lo schema, per quanto ben strutturato, non è però esaustivo, perché non mette in risalto alcuni aspetti abbastanza cruciali come le rappresentazioni e la capacità di previsione che pure giocano un ruolo fondamentale.

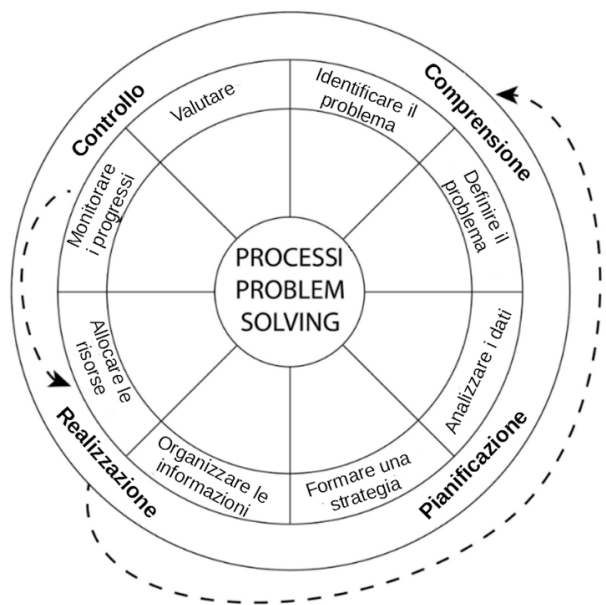

Figura 1. II problem solving come ciclo, tratto da Robertson (2016, p.18). 
Esiste infatti una relazione significativa tra le abilità visuo-spaziali e i risultati in matematica in generale e nel PS in particolare (Battista, 1990; Hegarty \& Kozhevnikov, 1999). Sebbene creare una corretta rappresentazione aiuti ad arrivare alla soluzione, va precisato che non tutti i tipi di rappresentazioni sono però d'aiuto. Sono stati individuati e distinti due tipi di rappresentazioni nel PS: una di tipo pittorico, ovvero raffigurazioni anche dettagliate della situazione come in un quadro o in una fotografia, ed un'altra di tipo schematico che tiene conto delle proprietà e delle relazioni tra gli oggetti che sono in gioco. Solo queste ultime correlano con buone performance nel PS (Hegarty \& Kozhevnikov, 1999).

\subsection{Le difficoltà nel problem solving e l'importanza della metacognizione}

A lungo si è indagato sui motivi che impediscono agli studenti dotati di buone capacità cognitive di ottenere performance altrettanto valide nel PS in matematica.

Nel suo corposo lavoro di indagine, Schoenfeld (1992), individua, tra le cause di insuccesso nel PS, la pratica di insegnanti e ricercatori nel ridurre la didattica esclusivamente agli aspetti cognitivi della performance, quando invece emerge con evidenza la necessità di una più ampia visione di ciò che la competenza comporta e dei fattori che influenzano la performance, tra i quali figura la metacognizione. Infatti, come spiega Schoenfeld (1992), questa include la consapevolezza dei propri punti di forza, dei punti deboli e dei processi ossia il possedere un repertorio di strategie ed azioni e il sapere come queste migliorino i risultati.

Cornoldi, nei suoi lavori (Cornoldi, 1995b; Cornoldi \& Lucangeli, 1997), sottolinea l'innovazione concettuale portata dal modello metacognitivo che ha consentito di mettere in luce come il successo in matematica dipenda anche dall'atteggiamento nei confronti della disciplina e come questo riguardi non solo l'alunno, ma anche il sistema scuola-famiglia con cui interagisce.

Negli ultimi decenni, molteplici sono gli studi che hanno messo in relazione la capacità di risolvere problemi matematici con lo sviluppo metacognitivo, in particolare in termini di una stretta correlazione tra i due: maggiori sono le capacità di uno studente di attivare la metacognizione, con l'utilizzo consapevole di strategie nella risoluzione di un problema, maggiore è la capacità di arrivare alla soluzione corretta (Schneider \& Artelt, 2010). Numerosi studi di intervento hanno dimostrato che studenti con performance di livello standard oppure con prestazioni matematiche particolarmente basse, traggono un vantaggio sostanziale da procedure di didattica metacognitiva. Infatti, gli alunni con difficoltà di apprendimento sono frequentemente affetti da carenze nella metacognizione che li portano al fallimento in una o più fasi del processo di PS, dalla mancata comprensione del testo del problema, all'identificazione delle informazioni rilevanti, delle operazioni e degli errori, alle difficoltà nella costruzione di una rappresentazione (Hutchinson, 1993; Judd \& Bilsky, 1989; Parmar, 1992; Xin, Jitendra \& Deatline-Buchman, 2005).

Da questo quadro risulta evidente quanto sia importante raccogliere I'appello degli studiosi rivolto agli insegnanti ad approfondire le proprie conoscenze sull'importanza della metacognizione nel PS al fine di progettare azioni efficaci e mirate allo sviluppo di questa competenza negli studenti.

\section{Obiettivi e logica dell'esperienza didattica}

L'obiettivo primario del lavoro di ricerca è stato quello di progettare e sperimentare un percorso didattico per migliorare le performance nel PS di studenti del terzo anno della scuola secondaria di primo grado e che fosse particolarmente improntato allo sviluppo degli aspetti metacognitivi, non solo riferiti alla conoscenza metacognitiva ma anche ai processi di regolazione e controllo che vengono messi in atto nella risoluzione di problemi. 
Un presupposto di partenza per la progettazione è stata l'osservazione che per sviluppare la metacognizione fosse fondamentale favorire la verbalizzazione dei processi risolutivi tramite il lavoro collaborativo a piccoli gruppi (Schoenfeld, 1989). L'importanza dell'interazione sociale nello sviluppo della metacognizione è assunta a fatto ben noto agli esperti del campo (Flavell, 1979; Schraw, Crippen \& Hartley, 2006; Cross \& Paris, 1988; Whitebread et al., 2009). II lavoro collaborativo non è tuttavia garanzia di riuscita di per sé, un ruolo chiave deve essere svolto dall'insegnante che, per garantire il buon funzionamento dei gruppi, interviene con tempi e modalità adeguate (Goos, Galbraith \& Renshaw, 2002).

Altra premessa riguarda l'importanza di fornire agli studenti una base di conoscenze sul processo risolutivo attraverso interventi didattici mirati. L'importanza di una didattica specifica per il PS è una proposta ormai decennale da parte della ricerca anglosassone, nella quale si sono sviluppate diverse tipologie di approccio al PS, come ad esempio la general-system theory (GST) (si veda per esempio Skyttner, 2005) o la schema-based instruction (SBI) (si veda per esempio Marshall, 2012). Nel nostro caso, abbiamo voluto ispirarci proprio alla GST, la quale comporta il fornire un'istruzione specifica sulle fasi del processo risolutivo (del tipo "comprendi il problema, fai una rappresentazione, pianifica la risoluzione, controlla il risultato"). Per aiutare il processo di categorizzazione del problema, utile alla costruzione di una rappresentazione, la GST prevede anche un'istruzione sui diversi tipi di problemi, classificati di solito in base al tipo di processo risolutivo.

Nella progettazione, inoltre, si è tratta ispirazione in particolar modo dal lavoro di Lester che, sebbene ormai datato, risulta ricco di osservazioni e propone con dovizia di particolari lo sviluppo di tutti questi aspetti con idee originali e ancora attuali (Lester, 1989; Lester, Garofalo \& Kroll, 1989). Nel suo progetto su classi di $7^{\circ}$ grado, equivalenti alla nostra classe seconda di scuola secondaria di primo grado, egli struttura interventi di istruzione specifica sul PS basati sul lavoro a piccoli gruppi di livello omogeneo in cui l'insegnante svolge diversi ruoli a seconda delle situazioni:

- osservatore esterno del processo di risoluzione dei problemi, che non interferisce con il processo stesso, ma ricava informazioni sui propri studenti lasciandoli liberi di confrontarsi senza farli sentire giudicati;

- facilitatore (o tutor), che orienta lo studente verso il superamento delle situazioni di stallo semplicemente ponendo delle domande al momento giusto, aiutandolo ad avere maggiore consapevolezza delle fasi del processo di risoluzione e delle possibili strategie da selezionare;

- modello di buon problem solver, il quale risolve problemi alla lavagna pensando ad alta voce, evidenziando la non linearità ovvero la circolarità del processo risolutivo mostrando che è lecito percorrere una strada non corretta e tornare sui propri passi se ci si accorge che non funziona.

Nella selezione dei problemi, inoltre, Lester utilizza una loro suddivisione in tre tipologie principali:

- a ritroso, il problema comincia dalla situazione finale e bisogna risalire ad un'informazione iniziale;

- ricerca di uno schema, il problema è strutturato secondo una sequenza, uno schema che si ripete, che bisogna individuare per arrivare alla soluzione;

- per tentativi, si tentano diverse strade fino ad individuare quella corretta.

Saper individuare la categoria a cui appartiene un problema è un processo tipico della conoscenza metacognitiva poiché richiama intorno alla categoria tutta una serie di processi risolutivi di cui si ha già avuto esperienza e che sono stati immagazzinati in memoria, i quali suggeriscono possibili strategie per raggiungere la soluzione.

Per valutare i progressi nel PS lavorando sulla metacognizione, Lester sottopone agli studenti un test strutturato in entrata ed uno in uscita. Per valutare invece l'evoluzione dei processi metacognitivi online si affida al metodo del think aloud ampiamente utilizzato in ambito scientifico (Lester, 1989). Tale metodo richiede però molto tempo e risorse, l'intervento di esperti dedicati, lo studio di registrazioni, trascrizioni e l'analisi complessa di dati. Tutto questo rende la procedura di difficile applicazione per la valutazione dello sviluppo metacognitivo da parte degli insegnanti in maniera autonoma senza il supporto di esperti, di fatto riducendo ai docenti la possibilità di sfruttare questi 
strumenti scientificamente validati nella pratica didattica.

Fortunatamente nel 2012 Jacobse e Harskamp hanno pubblicato un lavoro in cui dimostrano che un metodo semplice da loro sviluppato e chiamato VisA correla nei suoi risultati con quelli ottenuti dal think aloud nell'analisi dei processi online di metacognizione nel PS. II metodo consiste nella somministrazione di un prediction test prima della risoluzione, con la richiesta di fare una previsione e di strutturare una rappresentazione del problema; e di un postdiction test alla fine del processo risolutivo che richiede di confermare o meno quanto affermato nel test di predizione. La valutazione si ottiene dall'analisi delle rappresentazioni, suddivise in pittoriche, semipittoriche e schematiche, insieme con il confronto tra il pre-test con il post-test (una descrizione più dettagliata del metodo è riportata in seguito, nella sezione sui materiali e metodi).

In aggiunta alla valutazione della metacognizione ottenuta con il VisA test, occorre valutare anche le altre due dimensioni che definiscono la competenza nel suo complesso, in accordo con il modello proposto da Castoldi, cioè quella cognitiva e quella relazionale (Castoldi, 2009).

Seguendo l'esempio di Lester, che nel suo progetto ha utilizzato test di verifica standardizzati su conoscenze e abilità per la valutazione della dimensione cognitiva, abbiamo utilizzato i test strutturati delle prove INVALSI di grado 8. La valutazione della dimensione relazionale è stata invece ottenuta con una rubrica appositamente sviluppata per l'osservazione (si veda in seguito la Tabella 1).

Infine, abbiamo ritenuto importante inserire nel progetto attività connotate dai criteri tipici della laboratorialità. La modalità laboratoriale prevede che i ragazzi facciano esperienze di soluzione di problemi reali o comunque derivanti dalla vita quotidiana utilizzando le conoscenze matematiche e collegandole con quelle acquisite in ambiti diversi, dimostrando quindi quali fra di esse siano diventate davvero loro patrimonio personale. La didattica laboratoriale cui facciamo riferimento in maniera specifica è quella del Problem Based Learning (PBL) (si veda ad esempio Padmavathy \& Mareesh, 2013), in cui la situazione problematica è la base del processo di acquisizione del sapere e, intorno a essa, vengono costruiti gli stimoli e gli strumenti per l'attività di risoluzione. La PBL è quindi una strategia didattica che organizza gli apprendimenti in matematica intorno all'attività di PS favorendo il pensiero critico, la presentazione di idee creative e la comunicazione matematica tra pari. Pertanto l'apprendimento nel PBL non avviene ascoltando, ma facendo, manipolando, ricercando e comunicando.

\section{Materiali e metodi}

Considerate le premesse e in accordo con il lavoro di Lester (Lester, 1989; Lester, Garofalo \& Kroll, 1989), nella progettazione dell'intervento abbiamo operato scelte metodologiche e didattiche, quali:

- lavoro a piccoli gruppi omogenei per livello, alternato a compiti individuali;

- momenti di riflessione nelle fasi precedenti e successive alla risoluzione;

- istruzioni agli studenti sulle fasi del PS e sulle tipologie di problemi;

- approccio laboratoriale;

- interpretazione di diversi ruoli da parte dell'insegnante.

L'intervento è stato condotto nelle tre classi dall'insegnante di matematica con il supporto del docente di tecnologia. Questa configurazione, già presente nel curricolo della scuola che prevede laboratori in compresenza di carattere tecnico-scientifico, è risultata adatta per favorire il confronto. Inoltre il gruppo dei sei docenti coinvolti si è incontrato a cadenza periodica per discutere l'andamento del percorso didattico.

Il campione è costituito dagli alunni di tre classi terze della scuola secondaria di primo grado; dall'analisi sono stati esclusi gli studenti che erano stati assenti il giorno della somministrazione di almeno 
una delle prove in entrata o in uscita; gli alunni con disabilità certificate (ma senza la condizione di gravità) hanno ugualmente partecipato al percorso ma i dati a loro relativi non sono stati tenuti in considerazione. L'analisi pertanto è stata effettuata su 54 studenti i quali sono stati così suddivisi in fasce di livello:

- 11 alunni di fascia BES (bisogni educativi speciali) cioè con difficoltà di apprendimento di vario tipo o con disturbi specifici dell'apprendimento (DSA);

- 15 di fascia bassa ovvero studenti che hanno conseguito un punteggio inferiore a 6 decimi nel test cognitivo in entrata;

- 28 di fascia alta ovvero studenti che hanno conseguito una valutazione al test cognitivo in entrata uguale o superiore ai 6 decimi.

Per definire meglio i gruppi ci siamo riferiti anche alle conoscenze degli insegnanti di classe riguardo alle caratteristiche dei propri alunni, sebbene il test d'entrata sia riuscito ottimamente a discriminare i livelli di performance nel PS noti di ciascun studente. In tale test nessuno degli alunni con BES ha ottenuto una votazione superiore a 6 decimi.

Successivamente, gli alunni sono stati organizzati in gruppi di lavoro omogenei di 4 o 5 persone.

Prima e dopo la risoluzione di un problema è stato somministrato il VisA test, consistente esattamente nella scheda di prediction (Figura 2) e nella scheda di postdiction (Figura 3) di Jacobse e Harskamp (2012, p. 141), con l'aggiunta nel post-test di alcune domande tratte dalla scheda di autovalutazione di Lester $(1989$, p. 32) in cui si chiede di individuare, per ogni problema, la tipologia di appartenenza, di definire il grado di difficoltà e di stimolare la conoscenza metacognitiva ricercando comunanze tra il problema e quelli incontrati in precedenza.

Con riferimento alla Figura 2, agli studenti veniva chiesto di colorare il semaforo di verde se pensavano di riuscire a risolvere il problema, di rosso se ritenevano che non sarebbero stati in grado di arrivare alla soluzione, di giallo se non erano in grado di fare una valutazione. Sul retro della scheda veniva poi richiesto di provare a fare una rappresentazione del problema che potesse aiutarli nella risoluzione. Al termine, su un'altra scheda, gli studenti dovevano colorare nuovamente il semaforo a seconda che fossero o meno stati in grado, secondo loro, di arrivare alla soluzione. Di seguito veniva chiesto di rispondere ad altre domande per favorire la riflessione, ma la risposta non influiva sulla valutazione della metacognizione (Figura 3).

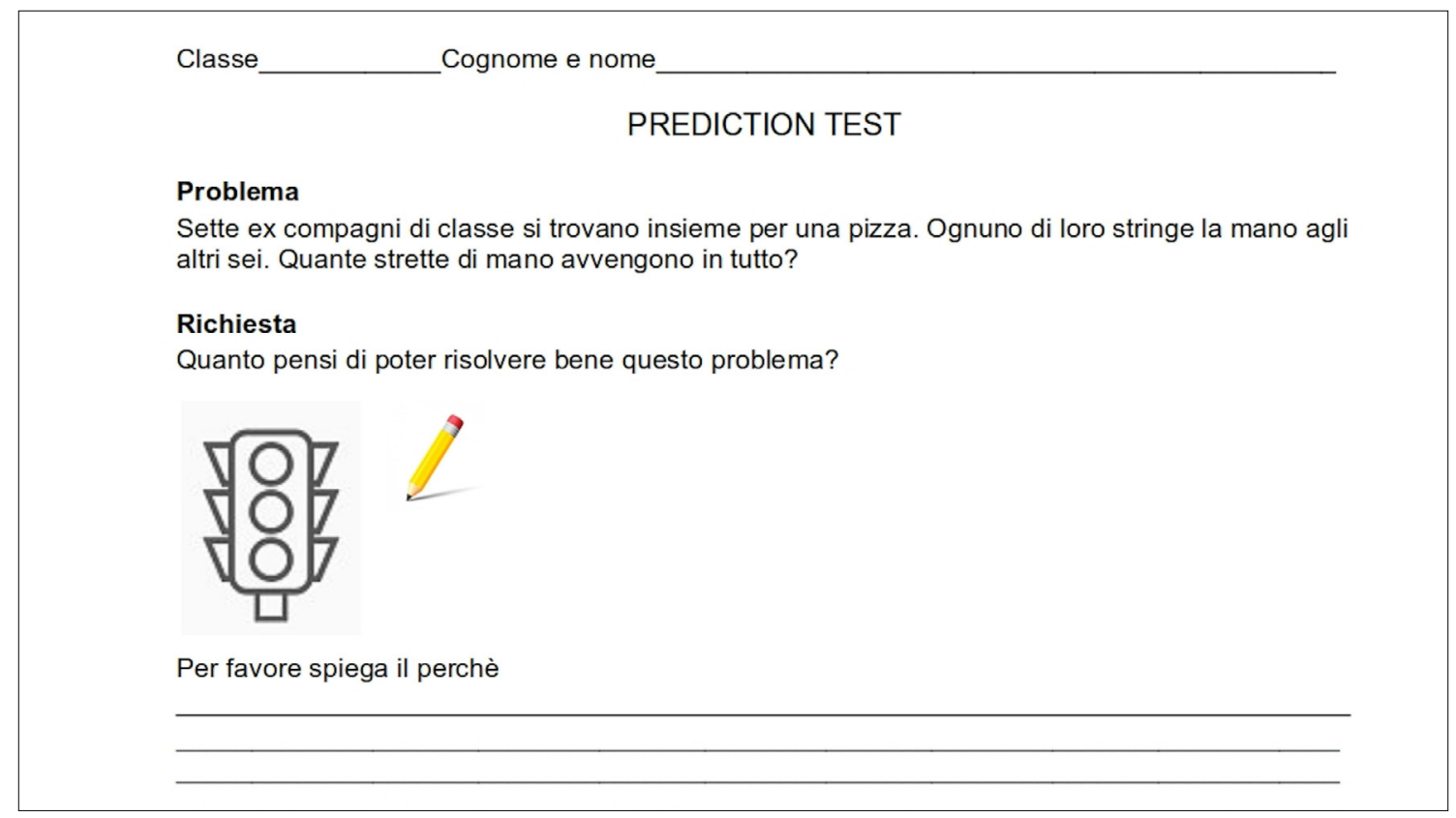

Figura 2. Esempio di scheda con il prediction test, utilizzata per la valutazione della metacognizione. 


\section{POSTDICTION TEST}

Pensa al problema su cui hai appena lavorato e poi cerchia la risposta che corrisponde a ciò che pensi:

1. Quanto sei sicura/o che la tua soluzione sia corretta?

ASSOlutamente ABbastanza NON MOLTO POCO PER NIENTE

2. Quanto è stato difficile per te questo problema?

ASSOLUTAMENTE ABBASTANZA NON MOLTO POCO PER NIENTE

3. Rispondi a questa domanda solo se pensi che la tua risposta sia giusta: perchè questo problema è stato facile per te?

4. Rispondi a questa domanda solo se pensi che la tua risposta sia sbagliata: perchè questo problema è stato difficile per te?

5. Hai mai risolto un problema come questo? Se è così puoi descriverlo?

Figura 3. La scheda per la riflessione metacognitiva (postidiction test) somministrata a risoluzione ultimata, ispirata al lavoro di Lester (1989).

Le conoscenze sul processo di PS e sulla tipologia di problemi sono state fornite tramite spiegazione frontale e discussione.

Per sviluppare il controllo metacognitivo sono state svolte attività che aiutassero ad individuare gli atteggiamenti efficaci o meno del problem solver. L'insegnante in alcune occasioni ha svolto il ruolo di modello: mentre risolve un problema pensando ad alta voce, mostra che anche un buon problem solver commette errori, li individua e li corregge mettendo continuamente in atto meccanismi di monitoraggio e controllo.

In particolare, è stato proposto un video ${ }^{2}$ preparato dagli insegnanti in cui il protagonista risolve un problema commettendo diversi errori: I'utilizzo di un cattivo problem solver, esterno alla relazione studente-insegnante, favorisce l'attenzione verso l'errore perché aiuta a percepirlo come un fenomeno intrinseco al processo di risoluzione anziché del soggetto.

Infine, poiché la scuola Don Milani nella sua costituzione sperimentale è promotrice della didattica laboratoriale per l'apprendimento, ci è sembrato coerente con la nostra esperienza l'inserimento nel percorso di una parte espressamente laboratoriale che contemplasse la risoluzione di problemi concreti con la manipolazione di oggetti reali e uso di strumenti di misura (vedi par. 5, descrizione del percorso).

\subsection{Monitoraggio e valutazione degli aspetti cognitivi, metacognitivi e relazionali}

Per poter apprezzare la valutazione del livello cognitivo e metacognitivo raggiunto dopo l'intervento didattico, si sono utilizzati VisA test e prove standardizzate in ingresso e in uscita e poi confrontate. Nella scelta del test cognitivo, abbiamo ricercato tra le prove strutturate di riferimento nazionale, una batteria di test standardizzata con indicatori misurabili per la valutazione, che fosse in accordo con le Indicazioni Nazionali per il curricolo della scuola dell'infanzia e del primo ciclo d'istruzione (MIUR, 2012). Esercizi o problemi aperti e situati, che attivano la competenza di PS e che sono documentati per permettere l'identificazione dei differenti processi messi in atto, si trovano in un'ampia raccolta

2. II video è disponibile sul canale YouTube della scuola: https://youtu.be/oVASVpVMZnY. 
nelle prove nazionali INVALSI. In particolare, in un utilissimo lavoro di sintesi dei documenti di valutazione delle prove internazionali sugli apprendimenti in matematica, Boero (2015) descrive come I'INVALSI abbia sviluppato un raggruppamento dei processi coinvolti nelle competenze matematiche, alle quali fanno riferimento i traguardi delle Indicazioni Nazionali (INVALSI, 2018b), secondo tre macro-aree denominate: conoscere, risolvere problemi, argomentare. Per quanto i tre processi non si possano attuare in modo indipendente I'uno dagli altri ma risultino intrecciati, nella documentazione INVALSI viene indicato dagli autori il processo prevalente in ogni quesito delle prove (INVALSI, 2015, 2016a). Per il test cognitivo in entrata abbiamo utilizzato la prova INVALSI di matematica per l'esame della classe terza della scuola secondaria di primo grado del 2015 e come test d'uscita abbiamo utilizzato I'edizione del 2016. La scelta di questi due test tra tutti quelli disponibili (non Computer Based, quindi fino al 2017) è stata casuale.

Per I'analisi abbiamo considerato la suddivisione suggerita da INVALSI separando i quesiti nei quali il processo risolutivo era prevalentemente di PS da quelli focalizzati sulle conoscenze oppure sull'argomentazione. Sono stati esclusi dai test i quesiti riguardanti argomenti non ancora trattati in classe.

Successivamente è stato eseguito un controllo incrociato per verificare la rigorosità delle valutazioni: i quesiti sono stati corretti dalla coppia dei docenti di classe e rivisti da un'altra coppia del gruppo di lavoro.

Nel VisA test per la valutazione della metacognizione, la procedura di calcolo del punteggio è studiata per essere semplice e veloce ed avviene su tre livelli:

1. Giudizio di predizione - se il giudizio di predizione dell'alunno è corretto (ad esempio, gli studenti predicono che risolveranno correttamente il problema e poi lo fanno; oppure che non sono in grado di risolvere il problema e poi sbagliano la risposta) ottengono 1 punto. Se la predizione è incerta (semaforo giallo) o sbagliata (ad esempio, prevedono che risolveranno il problema correttamente e poi non lo fanno; oppure predicono che non sono in grado di risolvere il problema e poi lo risolvono correttamente) ottengono 0 punti.

2. Visualizzazione del problema - gli studenti ottengono 0 punti se hanno fatto una rappresentazione pittorica non utile alla risoluzione del problema; 0,5 punti per rappresentazioni che sono in parte pittoriche ed in parte schematiche o con caratteristiche matematiche; 1 punto per le rappresentazioni schematiche utili alla risoluzione del problema.

3. Giudizio di post-dizione - il punteggio viene dato allo stesso modo del giudizio di predizione: 1 punto quando il giudizio a posteriori è corretto e 0 quando è sbagliato.

Per la valutazione degli aspetti relazionali, l'insegnante ha effettuato attività di osservazione al fine di stimare i livelli di partecipazione e di intervento dei propri studenti, utilizzando la rubrica in Tabella 1. Infine, anche il punteggio della metacognizione è stato poi convertito in decimi, in modo che il voto finale potesse essere calcolato facendo una media tra le valutazioni delle dimensioni cognitiva, metacognitiva e relazionale.

\begin{tabular}{|l|l|l|}
\hline Voto & Partecipazione & Interventi \\
\hline $9-10$ & $\begin{array}{l}\text { Ha partecipato sempre attivamente al lavoro di } \\
\text { gruppo dando un contributo fondamentale }\end{array}$ & $\begin{array}{l}\text { Ė intervenuto spesso per fare domande perti- } \\
\text { nenti o per contribuire alla discussione }\end{array}$ \\
\hline $7-8$ & Ha partecipato al lavoro di gruppo con costanza & $\begin{array}{l}\text { Ha fatto alcuni interventi pertinenti o ha fatto } \\
\text { domande }\end{array}$ \\
\hline $5-6$ & $\begin{array}{l}\text { Ha partecipato al lavoro di gruppo in modo non } \\
\text { costante o in maniera poco attiva }\end{array}$ & Raramente ha posto domande o fatto interventi \\
\hline$<5$ & Non ha quasi mai partecipato al lavoro & Non ha mai fatto interventi o posto domande \\
\hline
\end{tabular}

Tabella 1. Rubrica per l'osservazione semistrutturata della dimensione relazionale nella competenza del PS. 


\section{Descrizione del percorso}

Il percorso si è svolto in 9 incontri da 2 moduli da 55 minuti, sull'arco di circa 2 mesi. II primo e l'ultimo incontro sono stati dedicati alla somministrazione del test cognitivo strutturato iniziale e finale. La Figura 4 mostra una infografica riassuntiva degli incontri e la Tabella 2 raccoglie le diverse attività su cui si è lavorato.

Ogni risoluzione di problema è stata preceduta dalla lettura ad alta voce del testo da parte dell'insegnante e dalla compilazione del prediction test ed è stata seguita dalla compilazione del postdiction test, dalla presentazione dei risultati dei gruppi alla classe e dalla discussione collettiva. Inizialmente sono state anche lette e commentate le autovalutazioni degli studenti alla fine degli incontri.

In generale, per il lavoro dei gruppi sono stati preparati problemi supplementari per occupare i gruppi più veloci nell'attesa che anche quelli più lenti completassero i loro elaborati.

Al termine di ogni lezione sono stati assegnati dei problemi da risolvere a casa che sono stati corretti all'inizio della lezione successiva; in queste situazioni, l'insegnante si è proposto inizialmente come modello, risolvendo lui stesso i problemi, mentre successivamente ha lasciato agli studenti a turno la correzione seguendo in definitiva il modello della cognitive strategy instruction (Montague, Enders \& Dietz, 2011) che prevede l'acquisizione guidata e progressiva dell'autonomia da parte dello studente nell'uso delle strategie di autoregolazione.

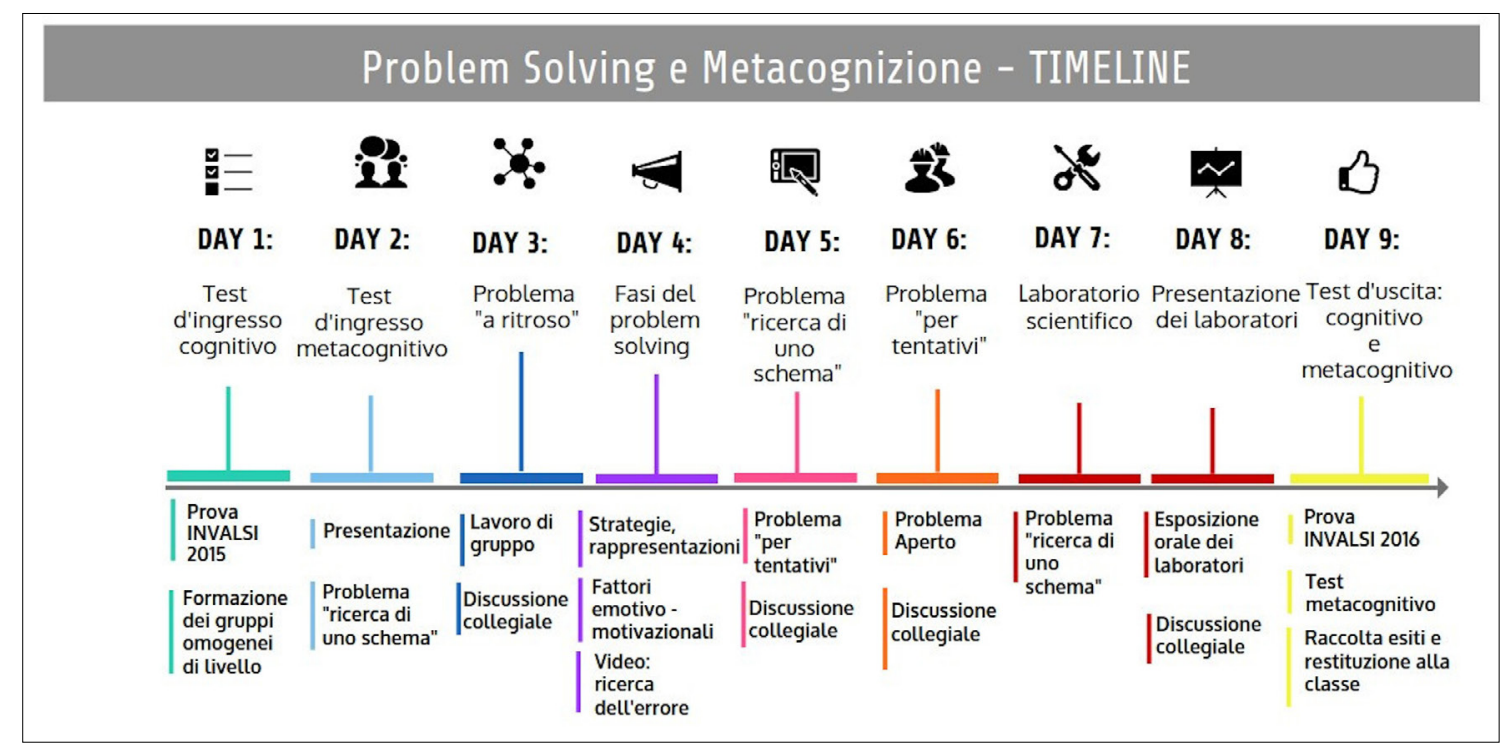

Figura 4. Timeline delle attività svolte con indicate le tipologie di problemi.

\begin{tabular}{|c|c|c|c|}
\hline \multicolumn{2}{|c|}{ Attività } & $\begin{array}{l}\text { Configurazione } \\
\text { degli alunni }\end{array}$ & $\begin{array}{l}\text { Ruoli degli inse- } \\
\text { ananti }\end{array}$ \\
\hline 1 & Test d'ingresso cognitivo & Individuale & Osservatore \\
\hline \multirow[t]{3}{*}{2} & Test d'ingresso metacognitivo (Figura 2) & Individuale & Osservatore \\
\hline & $\begin{array}{l}\text { Problema n. 1, Strette di mano: "Sette ex compagni di classe } \\
\text { si ritrovano insieme per una pizza. Ognuno di loro stringe } \\
\text { la mano agli altri sei. Quante strette di mano avvengono in } \\
\text { tutto?" }\end{array}$ & $\begin{array}{l}\text { Gruppi di livello } \\
\text { omogenei }\end{array}$ & Osservatore Modello \\
\hline & $\begin{array}{l}\text { Problema simile per compito e testo di autovalutazione } \\
\text { (Allegato 1) }\end{array}$ & Individuale & \\
\hline
\end{tabular}




\begin{tabular}{|c|c|c|c|}
\hline \multirow[t]{2}{*}{3} & $\begin{array}{l}\text { Problema n. } 2 \text { (a ritroso), La donna alla fonte: "Tanto tempo } \\
\text { fa nell'antica Cina, un'anziana donna si recava tutti i giorni a } \\
\text { prendere l'acqua al pozzo mettendosi sulle spalle un bastone } \\
\text { con alle estremità appesi due secchi. Ogni giorno riempiva } \\
\text { i secchi con } 6 \text { litri d'acqua ciascuno e lentamente tornava a } \\
\text { casa. Uno dei due secchi, però, essendo rotto, perdeva } 1 \text { dl } \\
\text { di acqua ogni } 100 \text { m e ogni volta che l'anziana donna torna- } \\
\text { va a casa trovava il secchio rotto pieno solo a metà. Quanto } \\
\text { era distante la casa dal pozzo?" }\end{array}$ & $\begin{array}{l}\text { Gruppi di livello } \\
\text { omogenei }\end{array}$ & Facilitatore \\
\hline & $\begin{array}{l}\text { Problema simile per compito e testo di autovalutazione } \\
\text { (Allegato 2) }\end{array}$ & Individuale & \\
\hline \multirow[t]{4}{*}{4} & Scheda di pre-visione (Allegato 3) & Individuale & \\
\hline & $\begin{array}{l}\text { Video No Problem con il Problema n. 3, Noleggio auto: } \\
\text { "Nadia affitta una macchina. La sua compagnia di noleggio } \\
\text { fa pagare } 50 \text { euro al giorno più } 0,40 \text { euro a chilometro } \\
\text { percorso. Pietro affitta una macchina in un'altra compagnia } \\
\text { di noleggio che fa pagare } 70 \text { euro al giorno più } 0,30 \text { euro a } \\
\text { chilometro. Per quanti chilometri dovranno guidare Nadia e } \\
\text { Pietro per pagare la stessa cifra?" }\end{array}$ & Individuale & $\begin{array}{l}\text { Facilitatore Osserva- } \\
\text { tore }\end{array}$ \\
\hline & Scheda di post-visione (Allegato 3) & Individuale & \\
\hline & Scheda di autovalutazione (Allegato 4) & Individuale & \\
\hline \multirow[t]{2}{*}{5} & $\begin{array}{l}\text { Problema n. } 4 \text { (per tentativi), La catena di anelli: "Devi ordi- } \\
\text { nare una catenina d'oro su internet per farne un braccialetto } \\
\text { a cui agganciare i } 6 \text { charms che ti sono stati regalati per il } \\
\text { compleanno. La catenina è costituita da anellini di } 10 \mathrm{~mm} \text { di } \\
\text { diametro che sono spessi } 1 \mathrm{~mm} \text { (come quelli rappresentati } \\
\text { in Figura 5) e il suo costo varia a seconda del numero di anelli } \\
\text { che la compongono. Supponendo che il vostro polso abbia } \\
\text { una circonferenza di } 15 \mathrm{~cm} \text { e considerando che i gancetti } \\
\text { per chiuderla sono lunghi in tutto } 2 \mathrm{~cm} \text {, da quanti anellini } \\
\text { dovrà essere composta la catenina da ordinare per spendere } \\
\text { il meno possibile? }\end{array}$ & Individuale & Modello \\
\hline & $\begin{array}{l}\text { Problema n. 5, La carovana nel deserto: "Una carovana è } \\
\text { bloccata nel deserto a } 6 \text { giorni di cammino dalla città, ogni } \\
\text { persona può portare con sé rifornimenti per } 4 \text { giorni. Qual è } \\
\text { il numero minimo di persone che devono partire per arrivare } \\
\text { in città e chiamare i soccorsi?" }\end{array}$ & $\begin{array}{l}\text { Gruppi di livello } \\
\text { omogenei }\end{array}$ & Facilitatore \\
\hline 6 & $\begin{array}{l}\text { Problema n. } 6 \text { (per tentativi), } 10 \text { sacchetti per } 10 \text { monete: } \\
\text { "Ho dieci sacchetti contenenti ciascuno dieci monete; in uno } \\
\text { di questi sono contenute monete di peso } 0,1 \mathrm{~g} \text { ciascuna, } \\
\text { nei rimanenti nove sono contenute monete da } 1 \mathrm{~g} \text { ciascuna. } \\
\text { Come possono individuare con una bilancia ad un solo piat- } \\
\text { to, con una sola pesata e senza l'aiuto di altri fattori in quale } \\
\text { sacchetto sono contenute le monete che pesano di meno?" }\end{array}$ & $\begin{array}{l}\text { Gruppi di livello } \\
\text { omogenei }\end{array}$ & Osservatore \\
\hline 7,8 & $\begin{array}{l}\text { Laboratorio di modellizzazione matematica di una legge di } \\
\text { fisica meccanica (Allegato 5) }\end{array}$ & $\begin{array}{l}\text { Gruppi di livello } \\
\text { omogenei }\end{array}$ & Facilitatore \\
\hline \multirow[t]{2}{*}{9} & Test d'uscita metacognitivo & Individuale & Osservatore \\
\hline & Test d'uscita cognitivo & Individuale & Osservatore \\
\hline
\end{tabular}




\subsection{Primo incontro}

Il primo incontro prevede la somministrazione del test di ingresso cognitivo (circa 75 minuti). Dagli esiti delle prove, gli insegnanti individuano gruppi di 4 - 5 alunni omogenei per fasce di livello.

\subsection{Secondo incontro}

L'insegnante introduce le attività presentando agli studenti le finalità e le tappe essenziali del percorso, rimarcando l'importanza nella risoluzione dei problemi del confronto verbale e della costruzione di rappresentazioni. Per evitare che la suddivisione nei gruppi omogenei possa incidere sull'autostima e l'autoefficacia di alcuni studenti fragili, è consigliabile rimarcare che assumerà particolare rilevanza positiva la propria e personale evoluzione.

L'insegnante consegna il testo del Problema n. 1, Strette di mano (Figura 2), senza spiegare e senza dare suggerimenti, quindi somministra il prediction test che questa volta funzionerà anche come test metacognitivo iniziale. Per 10 minuti, in silenzio, ogni alunno cerca una soluzione individuale del problema utilizzando qualunque rappresentazione (pittorica, schematica ecc.). L'insegnante al termine della compilazione ritira i prediction test.

Segue l'attività di PS che per i primi 10 minuti vede gli studenti, suddivisi in gruppi omogenei, affrontare una prima fase detta pencil down, in cui, senza poter scrivere, discutono e mettono insieme le idee per capire come risolvere il problema. Solo successivamente scrivono su un foglio e verificano i processi risolutivi ipotizzati dal gruppo.

Viene quindi effettuata la compilazione individuale del postdiction test e segue una discussione di classe e la raccolta dei risultati. Durante la discussione è importante che emerga l'importanza del costruire una rappresentazione del problema che sia effettivamente d'aiuto per arrivare alla soluzione. A questo scopo si possono confrontare le rappresentazioni proposte dai diversi gruppi per trovare quelle più efficaci.

Nella parte rimanente della lezione, ogni gruppo inventa il testo di un problema da sottoporre all'insegnante (completo di esecuzione e svolgimento) allo scopo di confrontare la propria strategia risolutiva con quella dell'insegnante che in questo caso funziona da modello.

Per compito a casa viene assegnata la risoluzione di un problema simile a quello svolto in classe e la redazione di un testo riassuntivo delle attività con autovalutazione (Allegato 1).

\subsection{Terzo incontro}

Dopo la correzione dei compiti per casa, l'insegnante, che svolge il ruolo di facilitatore, legge il testo del Problema n. 2, La donna alla fonte, del tipo a ritroso (Tabella 2) quindi consegna il prediction test. Alla fase di pencil down, segue la risoluzione del problema completa di rappresentazione. Al termine avviene la compilazione individuale del postdiction test e la condivisione delle soluzioni con la classe con esposizione dei risultati.

Ogni alunno scrive un sommario di ciò che è stato fatto, come compito a casa viene completato il sommario con l'autovalutazione e assegnati problemi a ritroso e trova uno schema (Allegato 2).

\subsection{Quarto incontro}

Si presentano agli alunni la classificazione dei tipi di processi risolutivi e le fasi principali del PS con particolare attenzione all'importanza delle rappresentazioni e all'influenza dei fattori emotivo-motivazionali sulla buona riuscita. Segue una discussione guidata dall'insegnante sulle fasi del processo di PS, sui diversi metodi di risoluzione. Alla luce della spiegazione si ripercorrono i problemi svolti suddividendoli nelle diverse categorie in base alle strategie di risoluzione e si rivedono le rappresentazioni fatte classificandole come utili o meno alla risoluzione del problema. 
Nella seconda parte della lezione, l'insegnante consegna una scheda con il testo del Problema n. 3, Noleggio auto, e la richiesta di leggerlo, provare una rappresentazione grafica e ipotizzare un possibile processo risolutivo (scheda di pre-visione nell'Allegato 3). In seguito, viene proiettato il video No problem $^{3}$ in cui il protagonista cerca di risolvere un problema commettendo però degli errori che gli alunni dovranno individuare e riportare (scheda di post-visione nell'Allegato 3). Segue il confronto tra il gruppo classe e l'insegnante, e la compilazione di una scheda di autovalutazione (Allegato 4).

\subsection{Quinto incontro}

L'insegnante si pone come modello esplicitando alla classe le decisioni e le azioni di regolazione per la risoluzione di un problema, permettendo così agli studenti di osservare le strategie di monitoraggio messe in atto da un esperto (processi online). A questo scopo viene risolto il Problema n. 4, La catena di anelli, del tipo per tentativi.

In seguito, I'insegnante chiede alla classe un commento riguardo al comportamento da esperto e dirige la discussione con le osservazioni degli alunni. L'insegnante vuole che gli studenti osservino che un buon problem solver non sempre va veloce e diretto alla soluzione, ma si pone domande su quello che sta facendo e pensando. Inoltre dimostra che disegnare degli schizzi e fare ipotesi sono tecniche legittime utilizzate anche da un esperto.

L'insegnante propone il Problema n. 5, La carovana nel deserto, del tipo trova uno schema chiedendo agli studenti, divisi nei soliti gruppi, di risolverlo ma pensando ad alta voce. Al termine sarà comunque I'insegnante a risolverlo alla lavagna ponendosi nuovamente come modello mentre i ragazzi compilano una scheda individuale di confronto, simile a quella del quarto incontro (Allegato 4).

\subsection{Sesto incontro}

Nel sesto incontro, gli alunni lavorano in gruppi al Problema n. 6, 10 sacchetti per 10 monete, del tipo per tentativi, durante il quale l'insegnante si dispone come osservatore. Gli studenti hanno a disposizione oggetti materiali da manipolare, anche se il problema nella sua particolare formulazione richiede agli studenti attenzione e cura nella fase di rappresentazione. Come di consueto gli alunni compilano i test di predizione e post-dizione.

\subsection{Settimo e ottavo incontro}

Nel laboratorio di scienze, gli studenti vengono chiamati a risolvere semplici problemi di modellizzazione di fisica meccanica (Figura 6). Ad ogni gruppo è affidato un set sperimentale (dinamometro con molla elastica, piano inclinato, bilancia a due bracci, modelli di leve) con la richiesta di studiare il comportamento dello strumento fisico al variare di alcune grandezze, di eseguire prove, formulare ipotesi e infine fare una rappresentazione descrittiva delle funzioni che legano le grandezze osservate (Allegato 5). Durante il laboratorio gli insegnanti si pongono come facilitatori, intervenendo solo se necessario. L'incontro successivo prevede il termine delle esperienze di laboratorio, la presentazione e il confronto dei risultati ottenuti dai gruppi alla classe e una discussione. 

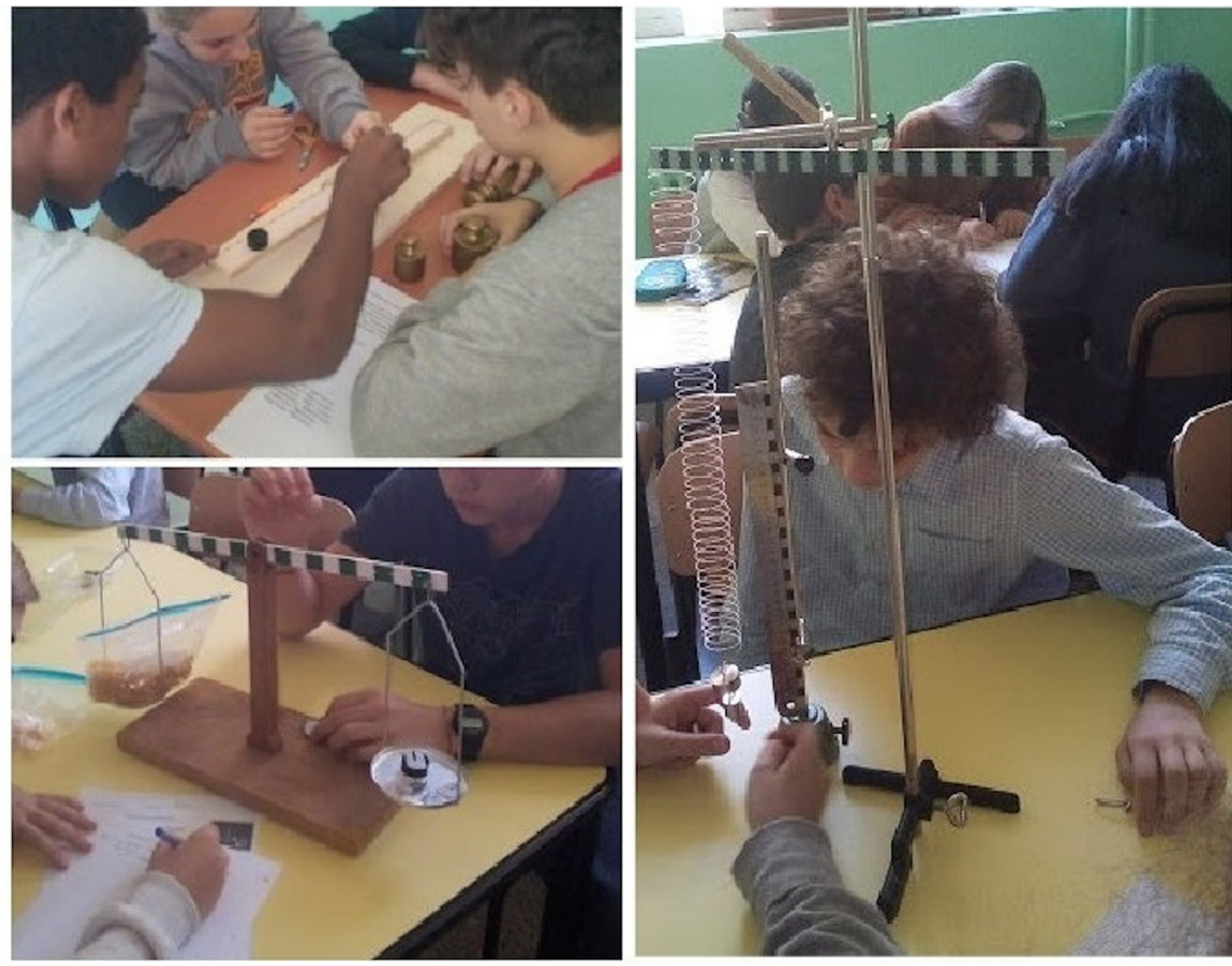

Figura 6. Alunni impegnati nelle attività di PS in un laboratorio di modellizzazione matematica su grandezze di fisica meccanica.

\subsection{Nono incontro}

Nell'ultimo incontro sono stati effettuati i test in uscita metacognitivo e cognitivo (circa 75 minuti).

\section{Risultati}

L'obiettivo principale dell'unità didattica è stato sperimentare una metodologia di lavoro che permettesse di potenziare o sviluppare negli alunni capacità metacognitive funzionali al miglioramento di performance nel PS matematico.

Durante tutto il percorso è stata via via restituita ai ragazzi l'evoluzione del proprio lavoro, grazie a discussioni di gruppo o individuali ed è stata esplicitata e condivisa la modalità di valutazione. Al termine si è potuto disporre di un modello di valutazione della competenza in modo completo, ovvero comprendente tutte e tre le dimensioni: cognitiva (test INVALSI), metacognitiva (VisA) e relazionale (osservazione semistrutturata); queste sono state inserite in una rubrica riassuntiva da cui, con una semplice operazione di conversione, sono state ricavate le valutazioni in decimi, quindi la valutazione sommativa è stata restituita agli alunni.

Dal confronto tra gli esiti ottenuti dai test in entrata e da quelli in uscita, si è potuta apprezzare una restituzione coerente con i nostri intenti: sul piano cognitivo è emerso un generale miglioramento riferito agli apprendimenti curricolari dei test standardizzati sebbene differenziato nelle varie fasce di livello. Sul piano metacognitivo, il miglioramento è stato più evidente e diffuso. 


\subsection{Risultati sulla metacognizione}

Nella Figura 7, sono riportati tutti i risultati dei punteggi ottenuti dal campione nel test VisA, visualizzati nel grafico a barre con due serie di dati a confronto (iniziale e finale). Dal grafico si nota, con buona evidenza, l'andamento positivo e in miglioramento della capacità metacognitiva dopo aver svolto il percorso progettato.

All'inizio del percorso la maggioranza degli alunni ottiene un punteggio basso, la moda infatti si colloca sullo 0 a dimostrazione di una capacità metacognitiva ancora scarsa. Anche 1 è un risultato molto frequente, mentre i punteggi 2, 2.5 e 3 sono raggiunti da solo un terzo degli alunni (18 su 54). La situazione finale è ben diversa, la moda si colloca sul 2 e i punteggi alti, dal 2 al 3, sono ottenuti stavolta dal 63\% degli alunni (34 su 54).

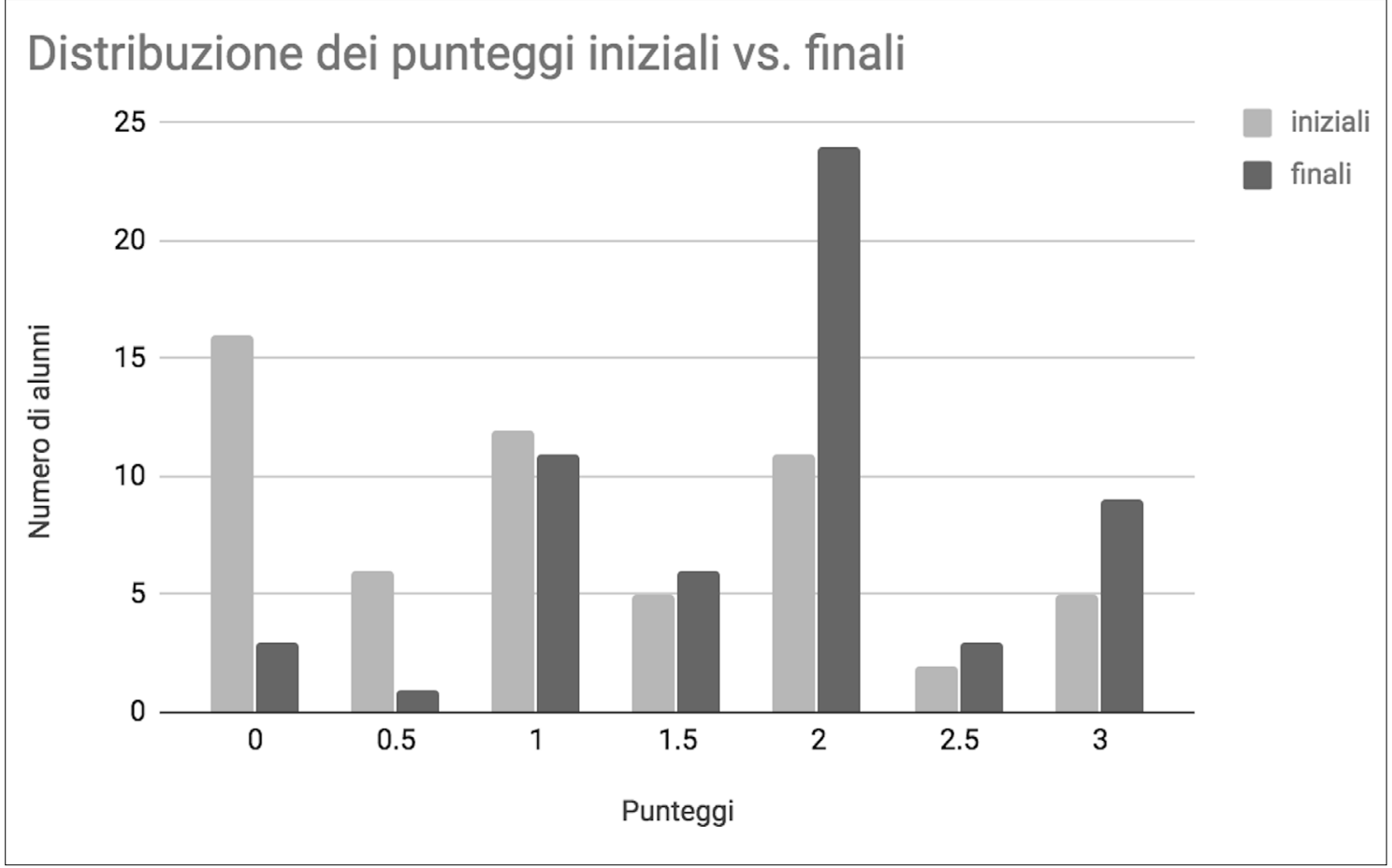

Figura 7. II diagramma a barre mostra la distribuzione in frequenza dei punteggi ottenuti dagli alunni nel test VisA iniziale (barre in grigio chiaro) e finale (barre in grigio scuro).

Nella Tabella 3, invece, i risultati del test VisA sono suddivisi in base alle fasce di livello, operazione che permette di apprezzare eventuali differenze nei sottogruppi.

Nella prima riga, in evidenza, sono riportati i risultati ottenuti sul totale degli alunni; nelle righe successive, la fascia alta, bassa e gli alunni con BES. Nelle colonne troviamo i punteggi riferiti alla situazione iniziale, finale e poi i dati che esplicitano il confronto con il T-test (terza e quarta colonna). I punti rappresentano la somma di tutti i punteggi ottenuti da tutti gli studenti della fascia considerata nel test iniziale (in entrata) o in quello finale (in uscita). I valori \% sul totale sono i punteggi ottenuti nel test VisA espressi in percentuale rispetto al punteggio massimo possibile (3 punti a studente). Nella colonna delle differenze, i punti sono ottenuti come differenza tra i punti finali e i punti iniziali; e analogamente sono state calcolate le differenze tra le percentuali. 


\begin{tabular}{|l|l|l|l|l|l|l|l|}
\hline & \multicolumn{2}{|l}{ Iniziale } & \multicolumn{2}{l|}{ Finale } & \multicolumn{2}{l|}{ Differenze $p=0,05$} & Migliorati \% \\
\cline { 2 - 8 } & Punti & $\%$ sul totale & Punti & $\%$ sul totale & Punti & \% sul totale & \\
\hline $\begin{array}{l}\text { Totale } \\
n=54\end{array}$ & 62,5 & $38,6 \%$ & 96 & $59,3 \%$ & $\begin{array}{l}+33,5 \\
p=0,0003^{*}\end{array}$ & $+20,7$ & 65,5 \\
\hline $\begin{array}{l}\text { Fascia alta } \\
n=28\end{array}$ & 41 & $48,8 \%$ & 60 & $71,4 \%$ & $\begin{array}{l}+19 \\
p=0,004^{*}\end{array}$ & $+22,6$ & 64,3 \\
\hline $\begin{array}{l}\text { Fascia bassa } \\
n=15\end{array}$ & 12 & $26,7 \%$ & 21,5 & $47,8 \%$ & $\begin{array}{l}+9,5 \\
p=0,01 *\end{array}$ & $+21,1$ & 66,7 \\
\hline $\begin{array}{l}\text { BES } \\
n=11\end{array}$ & 9,5 & $28,8 \%$ & 14,5 & $43,9 \%$ & $\begin{array}{l}+5 \\
p=0,13\end{array}$ & $+15,2$ & 54,5 \\
\hline
\end{tabular}

Tabella 3. Confronto tra situazione iniziale e finale del test VisA per la metacognizione.

Nella quarta colonna, la \% dei migliorati si riferisce alla percentuale di alunni che hanno migliorato la propria prestazione rispetto a quella iniziale, cioè coloro per i quali è verificata la seguente diseguaglianza:

$$
\text { (punteggio VisA finale - punteggio VisA iniziale) }>0
$$

Le differenze dei punti tra i due campioni sono state analizzate mediante un T-test a una coda con un livello di confidenza del $95 \%$. I dati con asterisco $\left({ }^{*}\right)$ mostrano una differenza significativa.

Dalla valutazione con il VisA test effettuata a fine percorso, gli studenti hanno dimostrato di aver migliorato sensibilmente la loro capacità di previsione della performance, ovvero hanno stimato con maggior confidenza la propria capacità o non capacità di risolvere il problema, anche se permaneva ugualmente una certa percentuale di indecisi (semaforo giallo). Solo in pochi hanno sbagliato la loro previsione (cioè hanno previsto di saper risolvere il problema e poi non I'hanno fatto o viceversa).

Di seguito riportiamo, a titolo di esempio, il problema usato per il test metacognitivo in entrata (VisA test). Come si nota in Figura 8, le rappresentazioni sono molto varie, alcune creative e non stereotipate; le prime tre in particolare, seppur dettagliate, non aiutano a trovare la soluzione che infatti è errata (42 strette di mano). II problema apparentemente semplice viene sottovalutato, infatti sono state raccolte diverse risposte nel prediction test del tenore «è un problema semplice, si deve fare solo un calcolo e sono in grado di farlo».

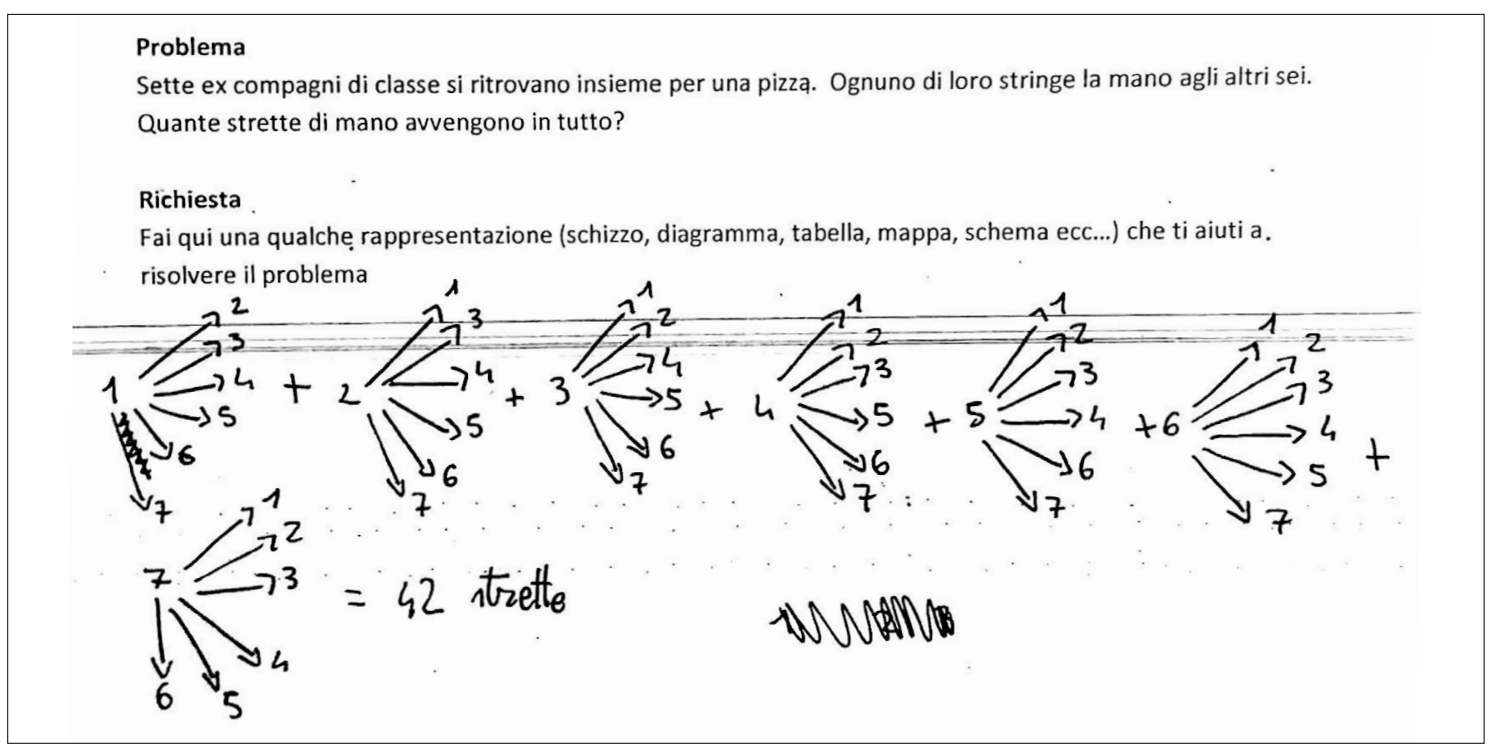


Sviluppare la metacognizione nel problem solving: un percorso di ricerca didattica nella scuola secondaria di primo grado/ Daniela Pietrapiana e Stefania Donadio
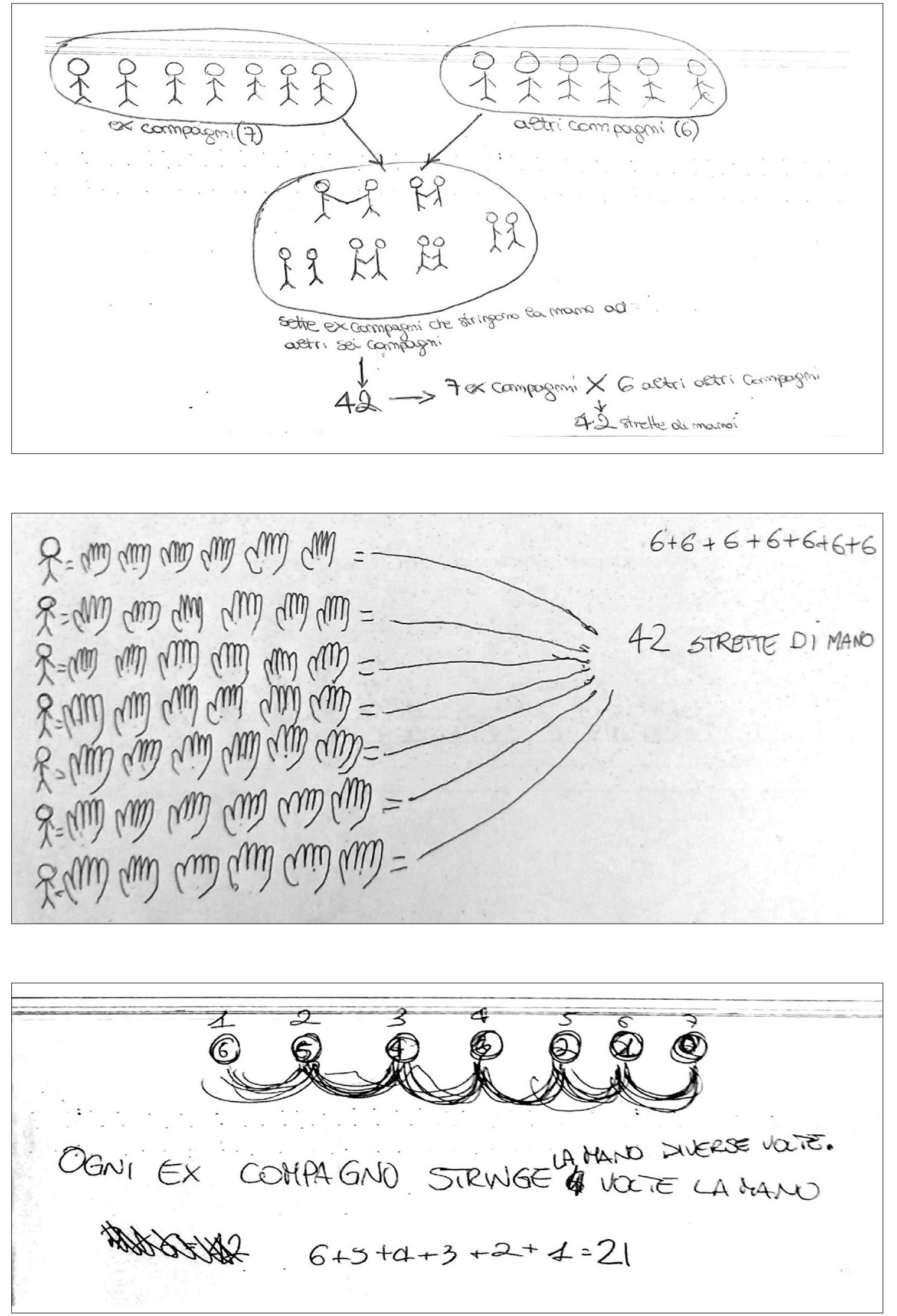

Figura 8a, b, c, d. Il testo del Problema n. 1, Strette di mano, con quattro esempi di rappresentazione grafica.

DdM 2020 (8), $115-140$

131 
Un esempio di evoluzione nella rappresentazione prodotta dagli alunni è riportato nelle figure seguenti, che raccolgono le soluzioni al Problema n. 2, La donna alla fonte, con le tre categorie di rappresentazioni: quelle ingenuamente pittoriche (Figura 9), semi-schematiche (Figura 10) e schematiche (Figura 11). Tutti i ragazzi in grado di produrre spontaneamente una rappresentazione schematica sono arrivati alla soluzione corretta, solo alcuni tra coloro che hanno utilizzato quelle del tipo semi-schematico vi sono riusciti, nessuno tra coloro che hanno utilizzato una rappresentazione pittorica è poi riuscito a risolvere il problema.

\section{Problema}

Tanto tempo fa nell'antica Cina, un'anziana donna si recava tutti i giorni a prendere l'acqua al pozzo mettendosi sulle spalle un bastone con alle estremità appesi due secchi. Ogni giorno rempiva i secchi con 6 litri d'acqua ciascuno e lentamente tornava verso casa. Uno dei due secchi, però, essendo rotto, perdeva $1 \mathrm{dl}$ di acqua ogni $100 \mathrm{~m}$ e ogni volta che l'anziana donna tornava a casa trovava il secchio rotto pieno solo a metà.

Quanto era distante la casa dal pozzo?

\section{Richiesta}

Fai qui una qualche rappresentazione (schizzo, diagramma, tabella, mappa, schema, etc) che ti aiuti a risolvere il problema.

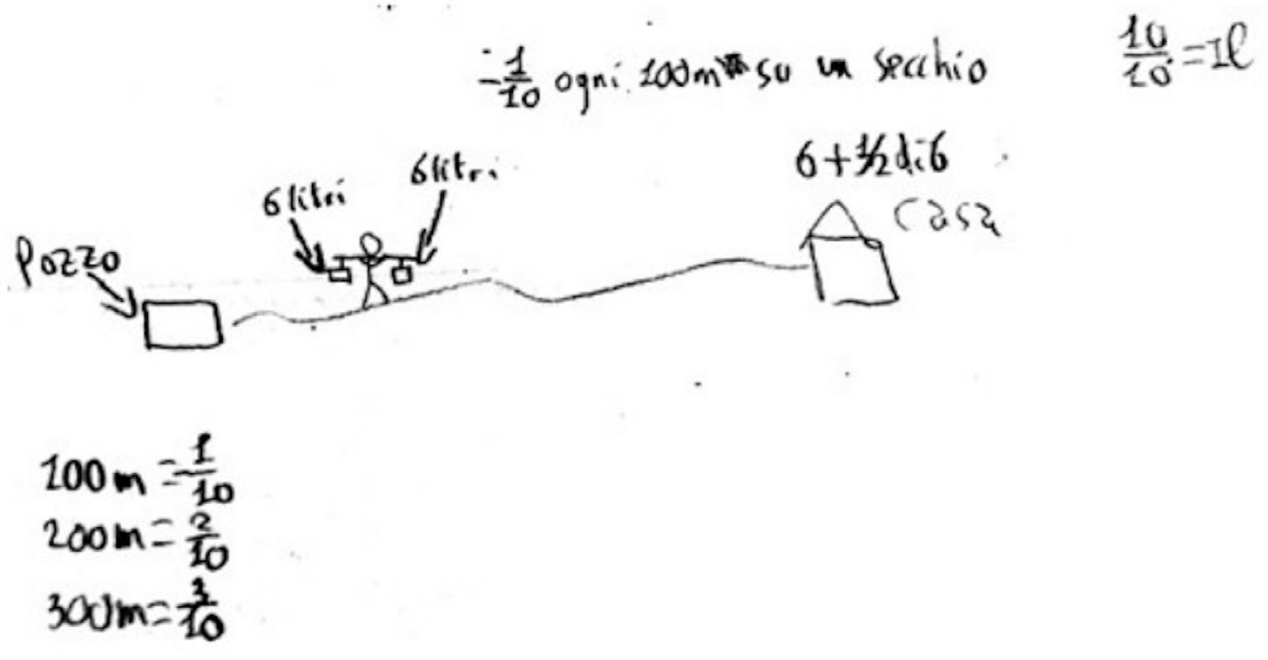

Figura 9. II Problema n. 2, della tipologia a ritroso, con testo della consegna e rappresentazione pittorica.

In Figura 9, si nota come la rappresentazione pittorica non aiuti a schematizzare il problema e sebbene I'alunno tenti anche un approccio di tipo numerico, questo è scollegato dalla rappresentazione e non gli permette di imbastire un procedimento risolutivo.

Le due rappresentazioni riportate in Figura 10 sono di tipo semi-schematico, infatti sebbene siano ancora presenti alcuni elementi pittorici (che probabilmente aiutano a creare una rappresentazione visiva della scena) questi sono disposti in una struttura più schematica; si noti la comparsa di elementi di astrazione come la definizione dell'unità di misura "goccia" e il tentativo di rappresentare la sequenzialità temporale degli eventi. 


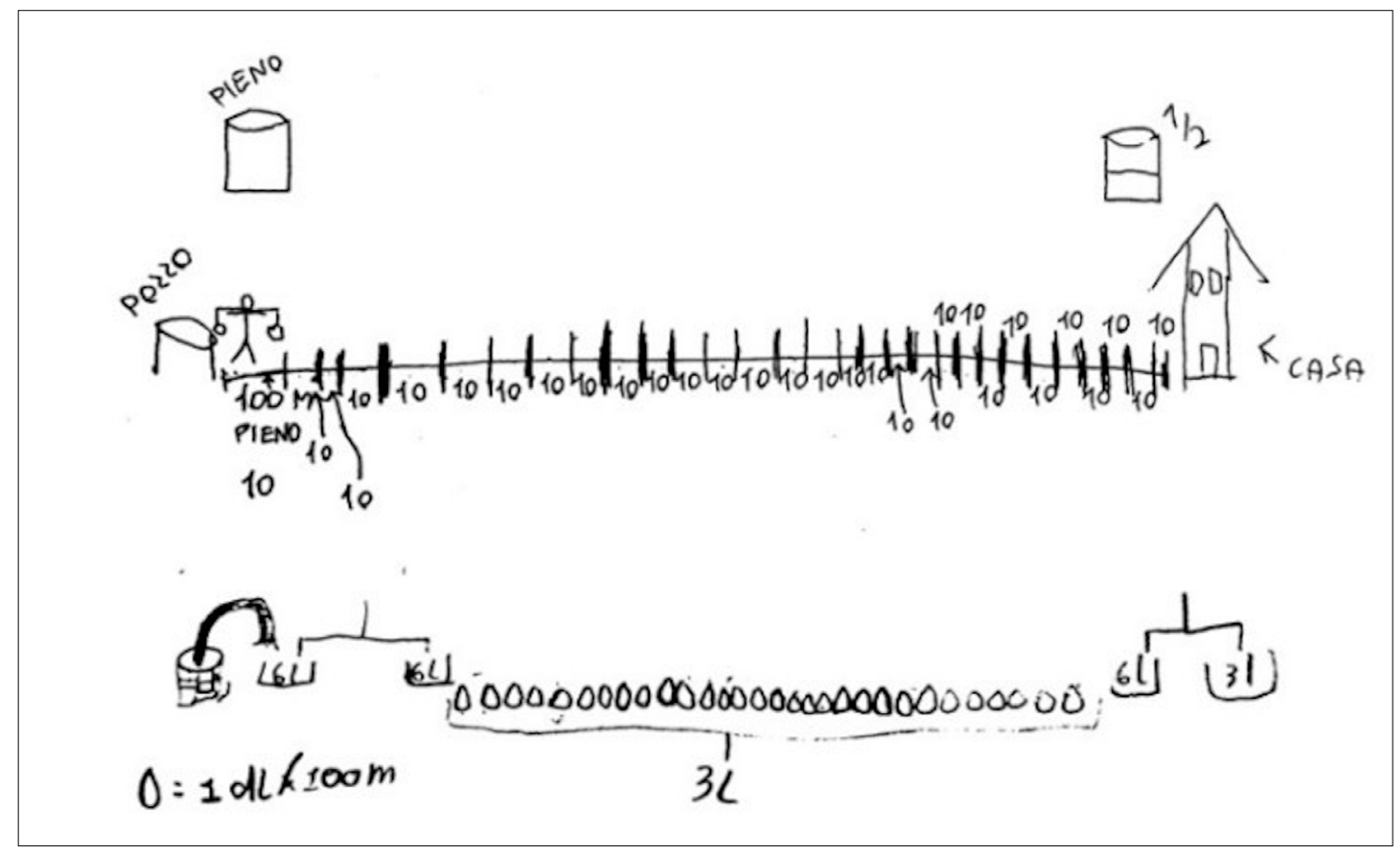

Figura 10. Due diverse rappresentazioni semi-schematiche del Problema n. 2 tentate dagli studenti.

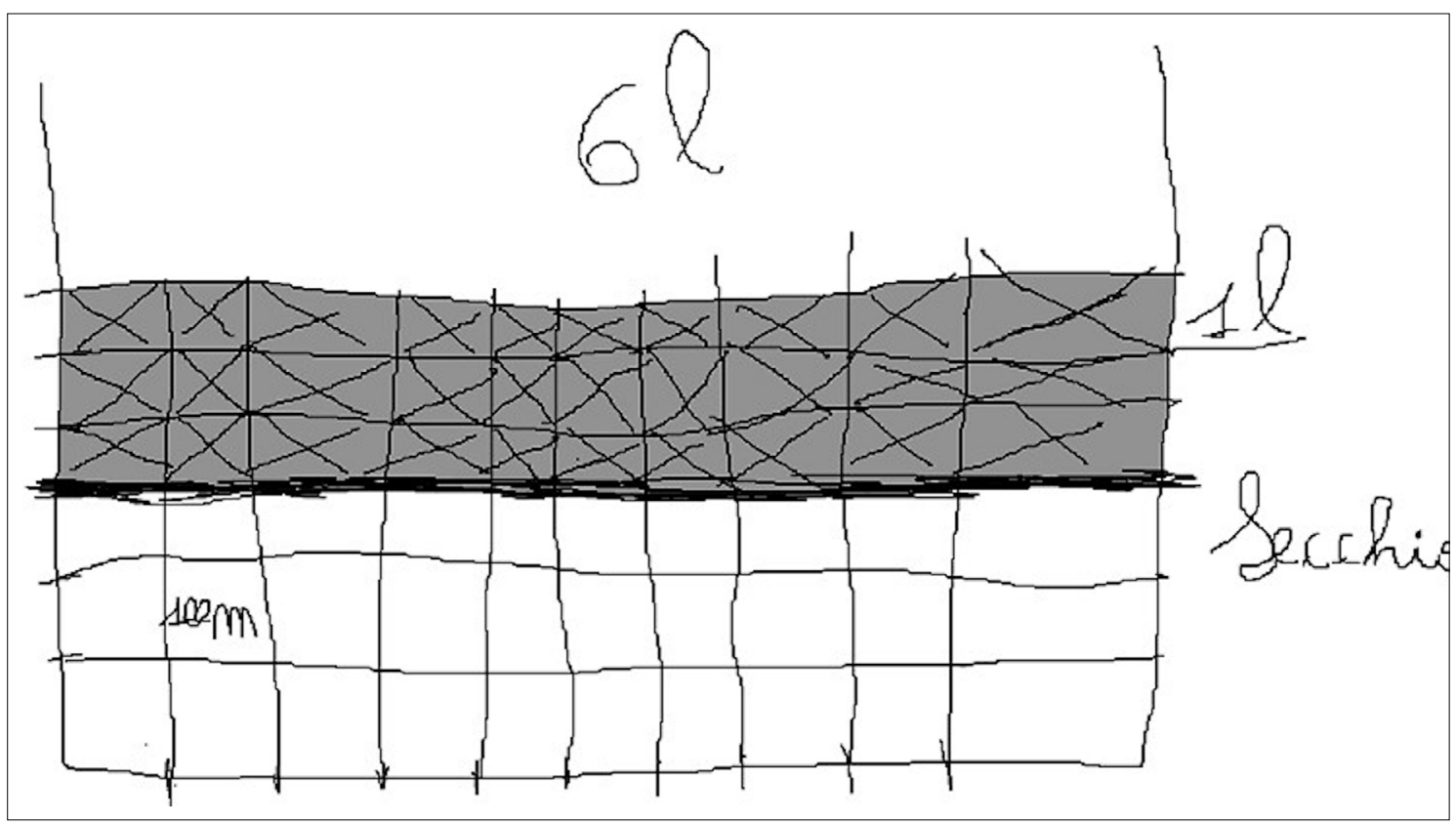

Figura 11. Rappresentazione schematica del Problema n. 2.

La Figura 11 tralascia tutti gli elementi narrativi della consegna e si concentra sull'elemento centrale del problema (il secchio che si svuota), rappresentandolo in maniera schematica con un modello geometrico. La soluzione corretta è stata raggiunta senza che l'alunno riportasse alcun calcolo numerico, ma è stata resa visibile nel disegno e si è ottenuta dal conteggio dei quadratini barrati (i litri di acqua che sono andati persi lungo la strada).

Nella Figura 12 si vede una soluzione del Problema n. 5, La carovana nel deserto, proposto a metà percorso: I'alunno è il medesimo che aveva utilizzato una rappresentazione pittorica con 42 manine 
nella Figura 8. In questo caso si nota che ha acquisito familiarità con la tipologia di problemi trova uno schema, infatti nella sua rappresentazione riproduce in colori diversi il pattern che ha individuato e cerca altresì di spiegare con cura apprezzabile il ragionamento che l'ha condotto alla soluzione, che è corretta.

Una carovana è bloccata nel deserto a 6 giorni di cammino dalla città, ogni persona può portare con sé rifornimenti per 4 giorni.

Qual è il $n^{\circ}$ minimo di persone che devono partire per arrivare in città e chiamare i soccorsi?

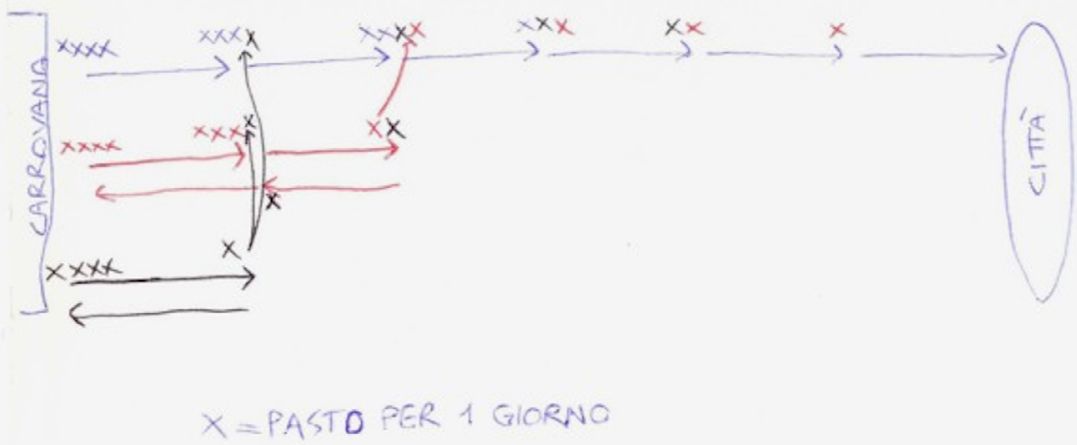

Occorrono tre persone: partono in tre, la sera del primo giorno una persona torna indietro e dà i suoi rifornimenti che avanzano agli altri due. II secondo giorno anche la seconda persona torna indietro e dà il rifornimento che gli avanza al terzo che cosi può arrivare in città.

Figura 12. Testo ed esempio di soluzione del Problema n. 4, La carovana nel deserto.

Quello che colpisce nel confronto tra le soluzioni costruite dallo stesso alunno nelle Figure 8 e 12 , è il passaggio da una rappresentazione pittorica molto legata alla realtà, quasi pratica, ad una più astratta e schematica.

Infine, nei laboratori di fisica meccanica conclusivi del percorso, tutti i gruppi sono riusciti a effettuare una rappresentazione formale della legge empirica modellizzata con l'utilizzo del piano cartesiano. Nella Figura 13 si vede un esempio di grafico piuttosto accurato, realizzato da un gruppo di alunni di fascia bassa.

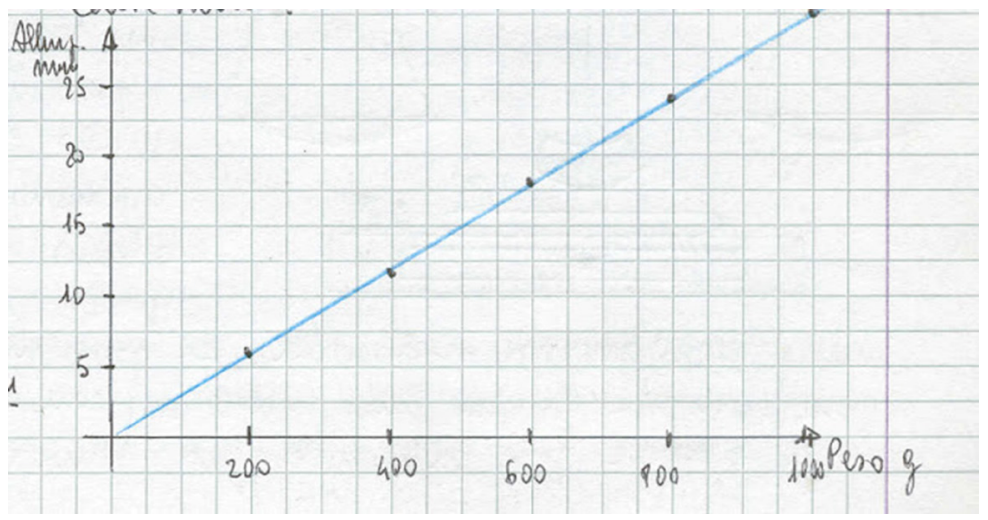

Figura 13. Rappresentazione grafica della soluzione di un problema di modellizzazione matematica di una legge empirica studiata in un laboratorio (allungamento di una molla elastica ottenuta dall'applicazione di masse differenti). 
Un alunno del gruppo che ha realizzato il grafico di Figura 13, riporta nella sua relazione:

«lo ero nel gruppo che doveva sperimentare l'allungamento di una molla elastica in relazione ai pesi che si potevano aggiungere o rimuovere. Dovevamo misurare la lunghezza della molla con i vari pesi e infine ricavare uno schema che dimostrasse la relazione tra allungamento e peso. L'esperimento mi ha permesso di capire che le due grandezze sono direttamente proporzionali».

Al termine del percorso molti più studenti sono riusciti ad utilizzare, nel test metacognitivo individuale in uscita, una rappresentazione schematica o semi-schematica, solo pochi ed in particolare appartenenti al gruppo dei BES hanno scelto una rappresentazione pittorica che non ha permesso loro di arrivare ad una soluzione corretta.

Riportiamo un esempio di restituzione da parte di due alunni ai quali è stata richiesta una considerazione conclusiva sul percorso svolto, il primo studente è di fascia alta e il secondo di fascia bassa: in entrambe emerge una buona consapevolezza di tipo metacognitivo.

G.: «Questo laboratorio è stato molto interessante e utile perché insegna come risolvere problemi utilizzando la logica. È stata bella anche la scoperta che ci sono diversi tipi di problemi e il fatto che non ci sia un unico metodo di risoluzione ma che ognuno di noi possa scegliere quello che preferisce perché più adatto al proprio modo di vedere le cose».

A.: «Dopo aver capito l'utilizzo e lo scopo del problem solving, mi sono accorto che molte volte utilizziamo questo tipo di procedura senza rendercene conto, perché, per esempio, nella traduzione dall'inglese all'italiano dobbiamo trovare delle strategie per analizzare la frase. Infatti per tradurre bisogna cercare il verbo, riordinare tutti i pezzi della frase e infine riscriverla in modo che in italiano risulti corretta. II laboratorio mi è piaciuto perché al posto di affrontare la solita lezione di matematica in classe siamo andati a svolgere un esperimento che ci ha visto utilizzare degli strumenti nuovi chiamati macchine semplici. Mi sono trovato bene perché mi piace lavorare in gruppo».

\subsection{Risultati del test cognitivo}

Come già detto, per la valutazione della componente cognitiva sono stati utilizzati i test INVALSI, I'edizione del 2015 in entrata e quella del 2016 in uscita, e sono stati analizzati separatamente i quesiti che avevano il PS come dimensione prevalente da quelli focalizzati su conoscere o argomentare.

Nella Figura 14, sono riportati i punteggi ottenuti dal campione sottoposto ai quesiti INVALSI sul PS, suddivisi in base alle fasce di livello (Alta, Bassa e BES) e aggregati su tutto il campione. I punteggi sono rappresentati con due serie di dati che riportano il valore medio ottenuto dal gruppo esaminato con una barra di errore che indica la deviazione standard, in grigio il punteggio in ingresso, in nero il punteggio in uscita. II confronto evidenzia l'andamento: un miglioramento per la fascia alta, bassa e nei dati complessivi, ma non in quella BES.

Come si vede dal grafico, il miglioramento è più evidente nella fascia bassa degli alunni, con circa un $19 \%$ di incremento dei punteggi, è più modesto per gli alunni di fascia alta (circa $6 \%$ ) e complessivamente si raggiunge il valore intermedio di circa il $12 \%$; nella fascia di alunni BES si osserva un andamento negativo nei risultati del $12 \%$. 


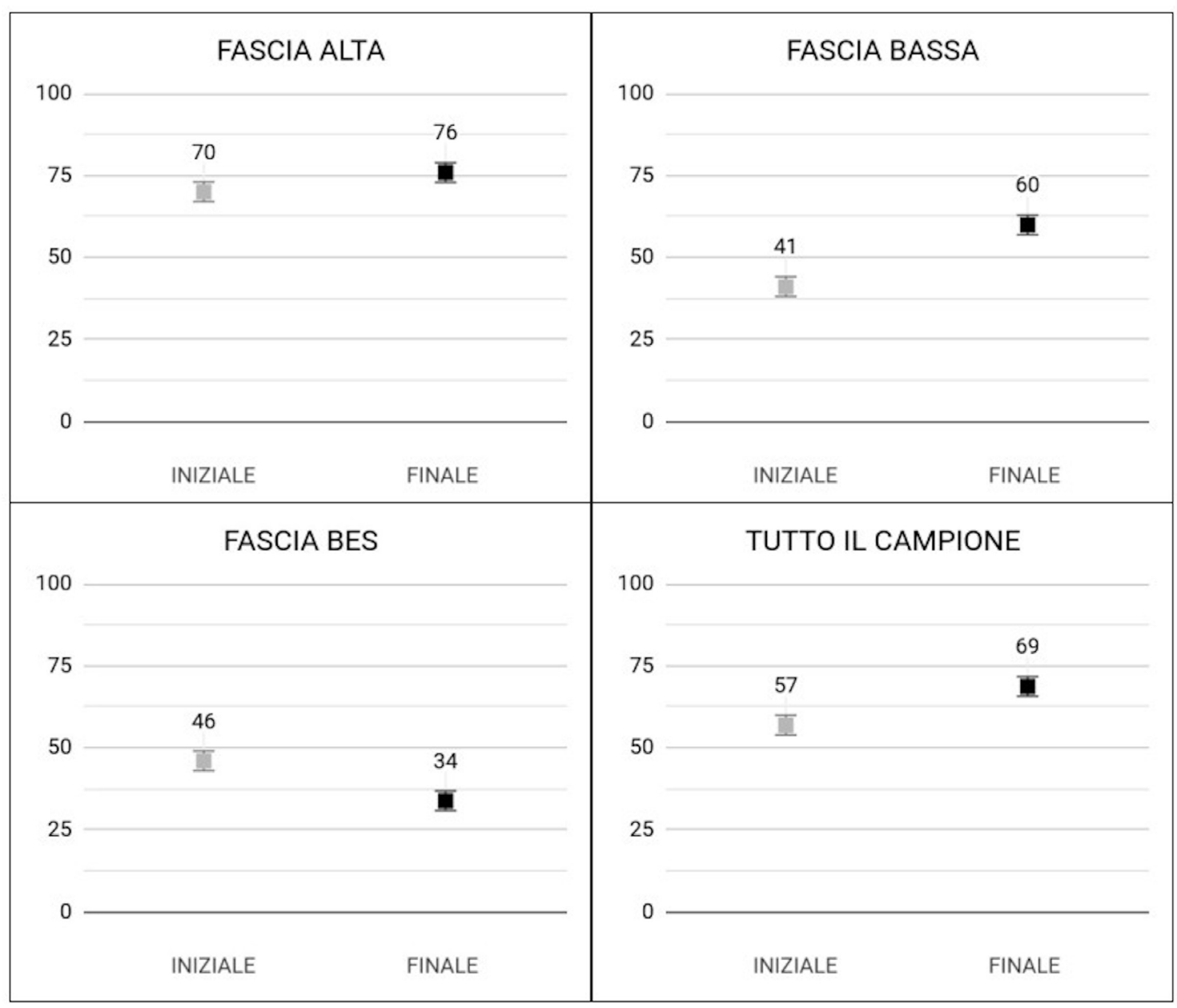

Figura 14. I punteggi medi riportati nei test INVALSI dal campione intero e dai sottogruppi: Fascia alta, Fascia Bassa, Fascia BES.

Nella Tabella 4 sono stati posti a confronto gli esiti dei quesiti con il PS come dimensione prevalente, rispetto a quelli con argomentare o conoscere, che sono stati in questo caso aggregati. Si è voluto così analizzare più in dettaglio se il miglioramento potesse riguardare in generale la competenza matematica oppure situarsi più specificamente nell'area del PS.

\begin{tabular}{|l|l|l|l|l|l|l|}
\hline & \multicolumn{2}{|l|}{ Problem Solving \% } & \multicolumn{2}{l|}{ Conoscere e argomentare \% } & \multicolumn{2}{l|}{$\begin{array}{l}\text { Confronto } \\
(p=0,05)\end{array}$} \\
\cline { 2 - 7 } & Iniziale & Finale & Iniziale & Finale & $\begin{array}{l}\text { Problem } \\
\text { Solving }\end{array}$ & $\begin{array}{l}\text { Conoscere } \\
\text { argomentare }\end{array}$ \\
\hline $\begin{array}{l}\text { Totale } \\
n=54\end{array}$ & 56,9 & 68,7 & 51,1 & 47,1 & $\begin{array}{l}+11,8 t \\
p=0,1 * *\end{array}$ & $\begin{array}{l}-4 t \\
p=0,25\end{array}$ \\
\hline $\begin{array}{l}\text { Fascia alta } \\
n=28\end{array}$ & 70 & 75,9 & 60 & 60 & $\begin{array}{l}+5,9 t \\
p=0,25\end{array}$ & $\begin{array}{l}0 t \\
p=0,50\end{array}$ \\
\hline $\begin{array}{l}\text { Fascia bassa } \\
n=15\end{array}$ & 41,1 & 59,9 & 45,3 & 41,4 & $\begin{array}{l}+18,8 t \\
p=0,025 *\end{array}$ & $\begin{array}{l}-3,9 t \\
p=0,30\end{array}$ \\
\hline $\begin{array}{l}\text { BES } \\
n=11\end{array}$ & 46 & 33,8 & 44,4 & 30 & $\begin{array}{l}-12,2 t \\
p=0,09 * *\end{array}$ & $\begin{array}{l}-14,4 t \\
p=0,02 *\end{array}$ \\
\hline
\end{tabular}

Tabella 4. Analisi delle differenze tra il test cognitivo in entrata e quello in uscita, suddiviso per le tre fasce di livello e totale (nelle righe) e per dimensione prevalente nei quesiti (nelle colonne). Nella colonna Confronto sono riportati gli esiti del T-test a una coda per la ricerca di differenze significative tra i due campioni in ingresso e in uscita. 
I valori rappresentano la media delle percentuali dei punteggi ottenuti in ciascun quesito usando la seguente formula:

$$
\text { \%PunteggioPerQuesito }=\frac{\left.\sum \text { (TotalePuntiQuesito }\right)}{\text { PunteggioMassimoOttenibile }} \cdot 100
$$

Le differenze tra i due campioni sono state analizzate mediante un T-test a una coda con un livello di confidenza del $95 \%$. I dati con * mostrano una differenza significativa, quelli con ** indicano una differenza significativa al $90 \%$ di confidenza.

Al termine del percorso, gli studenti hanno migliorato la propria prestazione nel test INVALSI nei quesiti a prevalenza di PS, mentre non vi sono variazioni significative per le altre tipologie di quesiti, che risultano peggiorare di pochi punti percentuali.

In particolare, gli studenti della fascia bassa, ovvero coloro che non avevano avuto esiti sufficienti nella prova in ingresso, migliorano significativamente la loro prestazione nel test d'uscita $(p=0,025)$. Anche gli studenti della fascia alta migliorano la propria performance, ma in maniera meno significativa, al 90\% di confidenza.

\section{Considerazioni conclusive}

È stato progettato e realizzato un percorso di ricerca didattica che ha avuto l'obiettivo di migliorare le capacità nel PS attraverso un potenziamento della metacognizione.

I docenti hanno potuto realizzare in totale autonomia tutte le fasi previste nel progetto, compresa la valutazione della metacognizione, solitamente considerato un aspetto critico che necessita l'intervento di esperti. Per raggiungere questo obiettivo, è stato indispensabile formare un gruppo di lavoro in cui tutti i docenti coinvolti si potessero confrontare in modo continuo e critico.

Al termine delle attività, è stato osservato un incremento generale e significativo dei processi metacognitivi legati al PS rilevati con il VisA test. I risultati ottenuti dal confronto tra i test cognitivi e metacognitivi in entrata ed in uscita evidenziano come ad un generale miglioramento delle capacità metacognitive abbia di fatto corrisposto quello della performance nel PS anche se non sempre in modo significativo.

La suddivisione in fasce di livello degli studenti ha permesso di mettere in risalto le differenze nell'efficacia del percorso a seconda del livello cognitivo di partenza. Sebbene sia gli studenti di fascia alta che quelli di fascia bassa abbiano mostrato un miglioramento nel PS, solo per questi ultimi l'incremento è stato significativo; ciò potrebbe essere dovuto al tipo di intervento che ha potuto agire su un margine di sviluppo prossimale più ampio per gli studenti di questo gruppo rispetto a coloro che partono già con un buon livello di competenza.

Inoltre, il percorso didattico non ha avuto influenze significative nelle capacità di risoluzione di quei quesiti in cui il PS non era il processo prevalente.

Una considerazione più dettagliata va condotta sulla scelta di prove cognitive di ingresso e di uscita differenti tra loro, che può comportare l'aggiunta di variabili nuove non necessariamente previste. Dall'analisi degli esiti pubblicata da INVALSI (INVALSI, 2018a, 2016b) è emerso che la prova INVALSI 2016 (utilizzata come test d'uscita) è più impegnativa di quella del 2015 (scelta per il test in entrata). Questo può spiegare la presenza di quesiti che hanno allargato la forbice degli esiti tra alunni con BES e senza, ma al tempo stesso ci ha rassicurati sull'efficacia del percorso visti gli esiti positivi degli altri gruppi.

Inoltre nel gruppo dei BES il percorso non sembra aver influito positivamente sulle capacità metacognitive. Questo dato non deve sorprendere per vari motivi: innanzitutto sono rientrati in questo 
gruppo studenti eterogenei per tipologia di difficoltà di apprendimento (dislessia, discalculia, problemi di comprensione linguistica ecc.) ma anche per differente strategia personale di compensazione, qualora attivata. Alunni così diversi tra loro necessitano di un tipo di didattica specifica e maggiormente individualizzata. Inoltre il gruppo BES era numericamente troppo piccolo per ottenere risultati maggiormente significativi.

In un ambito di ricerca azione, un miglioramento da apportare al percorso didattico è quello di renderlo maggiormente inclusivo, mediante l'utilizzo di metodologie di dimostrata efficacia per alunni con BES, inserendo ad esempio elementi tipici della schema-based instruction ed in generale della cognitive strategy instruction (Montague, Enders \& Dietz, 2011). Un'osservazione focalizzata sugli studenti con BES, inoltre, sarebbe utile per la pianificazione delle attività, per lo studio delle modalità di personalizzazione delle consegne e per indagare sulle strategie di risoluzione o compensazioni messi in atto. Il presente lavoro si presta a un possibile sviluppo che preveda le attività di PS pianificate durante tutto I'arco del triennio della scuola secondaria di primo grado, così da permettere una costruzione più strutturata e armonica della metacognizione e un monitoraggio più esteso. II progetto è in continua revisione e allo stato attuale è stato adattato per poter essere avviato già nelle classi prime.

\section{Bibliografia}

Battista, M. (1990). Spatial Visualization and Gender Differences in High School Geometry. Journal for Research in Mathematics Education, 21(1), 47-60.

Boero, P. (2015). Prove INVALSI: A proposito della definizione delle macro-aree di competenze, Scuola autori, INVALSI. Disponibile in: https://invalsi-areaprove.cineca.it/docs/autori/Presentazione Boero.pdf (consultato il 14.05.2020).

Brown, A. L. (1978). Knowing when, where, and how to remember: A problem of metacognition. In R. Glaser (Ed.), Advances in instructional psychology 1 (pp. 77-165). Hillsdale, N. J.: Erlbaum.

Brown, A. L., \& DeLoache, J. S. (1978). Skills, plans, and self-regulation. In R. S. Siegel (Ed.), Children's thinking: What develops? (pp. 3-35). Hillsdale, N.J.: Erlbaum.

Castoldi, M. (2009). Valutare le competenze, percorsi e strumenti. Roma: Carocci.

Castoldi, M. (2011). Progettare per competenze, percorsi e strumenti. Roma: Carocci.

Castoldi, M. (2013). Curricolo per competenze, percorsi e strumenti. Roma: Carocci.

Cornoldi, C. (1995a). Metacognizione e apprendimento. Bologna: II Mulino.

Cornoldi, C. (1995b). Matematica e metacognizione: atteggiamenti metacognitivi e processi di controllo, (Vol. 43). Trento: Erickson.

Cornoldi, C., \& Lucangeli, D. (1997). Mathematics and metacognition: What is the nature of the relationship?. Mathematical cognition, 3(2), 121-139.

Cross, D. R., \& Paris, S. G. (1988). Developmental and instructional analyses of children's metacognition and reading comprehension. Journal of Educational Psychology, 80(2), 131-142.

Desoete, A., Roeyers, H., \& De Clercq, A. (2003). Can offline metacognition enhance mathematical problem solving?. Journal of Educational Psychology, 95(1), 188-200. 
Flavell, J. H. (1976). Metacognitive aspects of problem solving. In L. B. Resnick (Ed.), The nature of intelligence (pp. 231-236). Hillsdale, NJ: Erlbaum.

Flavell, J. H. (1979). Metacognition and cognitive monitoring: A new area of cognitive developmental inquiry. American Psychologist, 34(10), 906-911.

Goos, M., Galbraith, P., \& Renshaw, P. (2002). Socially mediated metacognition: Creating collaborative zones of proximal development in small group problem solving. Educational Studies in Mathematics, 49(2), $193-$ 223.

Hegarty, M., \& Kozhevnikov, M. (1999). Types of visual-spatial representations and mathematical problem solving. Journal of Educational Psychology, 91(4), 684-689.

Hutchinson, N. L. (1993). Effects of cognitive strategy instruction on algebra problem solving of adolescents with learning disabilities. Learning Disability Quarterly, 16(1), 34-63.

INVALSI (2015). Servizio Nazionale di Valutazione a.s. 2014/15 Guida alla lettura Prova Nazionale al termine del primo ciclo: Matematica Classe terza - Scuola secondaria di I grado. Disponibile in: https://invalsi-areaprove. cineca.it/docs/attach/2015 guida L08 DICEMBRE.pdf (consultato il 16.06.2020).

INVALSI (2016a). Servizio Nazionale di Valutazione a.s. 2015/16 Guida alla lettura Prova Nazionale al termine del primo ciclo: Matematica Classe terza - Scuola secondaria di I grado. Disponibile in: https://invalsi-area prove.cineca.it/docs/file/2016-GUIDA-L08.pdf (consultato il 16.06.2020).

INVALSI (2016b). Rilevazioni Nazionali degli Apprendimenti 2014/15, rapporto risultati. Disponibile in: https:// www.invalsi.it/invalsi/doc evidenza/2015/034 Rapporto Prove INVALSI 2015.pdf (consultato il 14.05.2020).

INVALSI (2018a). Rilevazioni Nazionali degli Apprendimenti 2015/16, rapporto risultati. Disponibile in: https:// www.invalsi.it/invalsi/doc evidenza/2016/07 Rapporto Prove INVALSI 2016.pdf (consultato il 14.05.2020).

INVALSI (2018b). Quadro di riferimento delle prove di INVALSI matematica (ver. 30.08.2018). Disponibile in: https://invalsi-areaprove.cineca.it/docs/file/QdR MATEMATICA.pdf (consultato il 14.05.2020).

Jacobse, A. E., \& Harskamp, E. G. (2012). Towards efficient measurement of metacognition in mathematical problem solving. Metacognition and Learning, 7(2), 133-149.

Judd, T. P., \& Bilsky, L. H. (1989). Comprehension and memory in the solution of verbal arithmetic problems by mentally retarded and nonretarded individuals. Journal of Educational Psychology, 81(4), 541-546.

Lai, E. R. (2011). Metacognition: A literature review. Always learning: Pearson research report, 24.

Lester, F. K. (1989). The role of metacognition in mathematical problem solving: a study of two grade seven classes, a project of the Mathematics Education Development Center. Indiana University.

Lester, F. K., Garofalo, J., \& Kroll, D. L. (1989). Self-confidence, interest, beliefs, and metacognition: Key influences on problem-solving behavior. In D. B. McLeod \& V. M. Adams (Eds.), Affect and mathematical problem solving (pp. 75-88). New York, NY: Springer.

Marshall, S. P. (2012). Schema-Based Instruction. In N. M. Seel (Ed.), Encyclopedia of the Sciences of Learning. Boston, MA: Springer.

MIUR (2012). Indicazioni nazionali per il curricolo della scuola dell'infanzia e del primo ciclo di istruzione. Annali della Pubblica Istruzione, Numero Speciale. Firenze: Le Monnier.

Montague, M., Enders, C., \& Dietz, S. (2011). Effects of cognitive strategy instruction on math problem solving of middle school students with learning disabilities. Learning Disability Quarterly, 34(4), 262-272. 
Padmavathy, R. D., \& Mareesh, K. (2013). Effectiveness of problem based learning in mathematics. International Multidisciplinary e-Journal, 2(1), 45-51.

Parmar, R. S. (1992). Protocol analysis of strategies used by students with mild disabilities when solving arithmetic word problems. Diagnostique, 17(4), 227-243.

Pietrapiana, D. (2016). Developing Metacognition at School: a Learning Integrated Approach. In J. Benson (Ed.), Metacognition: theory, performance and current research (pp. 95-121). Hauppauge NY: Nova Science Publishers.

Pintrich, P. R., Wolters, C. A., \& Baxter, G. P. (2000). Assessing metacognition and self-regulated learning. In G. Schraw \& J. C. Impara (Eds.), Issues in the Measurement of Metacognition (pp. 43-97). Lincoln, NE: Buros Institute of Mental Measurements.

Robertson, S. I. (2016). Problem solving: perspectives from cognition and neuroscience. ( $2^{\circ}$ ed.) Psychology Press, Londra: Routledge Taylor \& Francis.

Schneider, W., \& Artelt, C. (2010). Metacognition and mathematics education. ZDM, 42(2), 149-161.

Schoenfeld, A. H. (1989). Ideas in the air: Speculations on small group learning, environmental and cultural influences on cognition, and epistemology. International Journal of Educational Research, 13(1), 71-88.

Schoenfeld, A. H. (1992). Learning to Think Mathematically: Problem Solving, Metacognition, and Sense-Making in Mathematics. In D. Grouws (Ed.), Handbook for Research on Mathematics Teaching and Learning (pp. 334-370). New York: MacMillan.

Schraw, G., Crippen, K. J., \& Hartley, K. (2006). Promoting self-regulation in science education: Metacognition as part of a broader perspective on learning. Research in science education, 36(1-2), 111-139.

Skyttner, L. (2005). General Systems Theory: Problems, Perspectives, Practice. London: World Scientific.

Veenman, M. V. J. (2005). The assessment of metacognitive skills: What can be learned from multi-method designs? In C. Artelt \& B. Moschner (Eds.), Lernstrategien und Metakognition: Implikationen für Forschung und Praxis (pp. 75-97). Berlin: Waxmann.

Veenman, M. V. J., Van Hout-Wolters, B. H., \& Afflerbach, P. (2006). Metacognition and learning: Conceptual and methodological considerations. Metacognition and learning, 1(1), 3-14.

Veenman, M. V. J., \& Van Cleef, D. (2019). Measuring metacognitive skills for mathematics: students' self-reports versus on-line assessment methods. ZDM, 51(4), 691-701.

Whitebread, D., Coltman, P., Pasternak, D. P., Sangster, C., Grau, V., Bingham, S., Almeqdad, Q., \& Demetriou, D. (2009). The development of two observational tools for assessing metacognition and self-regulated learning in young children. Metacognition and learning, 4(1), 63-85.

Xin, Y. P., Jitendra, A. K., \& Deatline-Buchman, A. (2005). Effects of mathematical word Problem-Solving instruction on middle school students with learning problems. The Journal of Special Education, 39(3), 181-192. 